Pacific

Journal of

Mathematics

HAUSDORFF DIMENSION OF LIMIT SETS FOR PARABOLIC IFS WITH OVERLAPS

K. Simon, B. Solomyak, and M. URbański 


\title{
HAUSDORFF DIMENSION OF LIMIT SETS FOR PARABOLIC IFS WITH OVERLAPS
}

\author{
K. Simon, B. Solomyak, and M. URbański
}

\begin{abstract}
We study parabolic iterated function systems with overlaps on the real line. We show that if a $d$-parameter family of such systems satisfies a transversality condition, then for almost every parameter value the Hausdorff dimension of the limit set is the minimum of 1 and the least zero of the pressure function. Moreover, the local dimension of the exceptional set of parameters is estimated. If the least zero is greater than 1, then the limit set (typically) has positive Lebesgue measure. These results are applied to some specific families including those arising from a class of continued fractions.
\end{abstract}

\section{Introduction.}

Let $\Phi=\left\{\phi_{1}, \ldots, \phi_{k}\right\}$ be a collection of self-maps on a closed interval $X \subset \mathbb{R}$. We call $\Phi$ an iterated function system (IFS). Under standard contractivity hypotheses, there is a unique non-empty compact set $J_{\Phi}$ such that $J_{\Phi}=\bigcup_{j=1}^{k} \phi_{j}\left(J_{\Phi}\right)$, called the limit set, or attractor, of the IFS.

If the sets $\phi_{j}(X)$ are mutually disjoint, then $J_{\Phi}$ is a Cantor set. If, in addition, $\phi_{j}$ are monotone, the limit set is known as a "cookie-cutter"; then it is more common to view $J_{\Phi}$ as the repeller of an expanding map $f: \bigcup_{j=1}^{k} \phi_{j}(X) \rightarrow X$ defined by $f(x)=\phi_{j}^{-1}(x)$ for $x \in \phi_{j}(X)$. Suppose that all the maps $\phi_{j}$ are in $\mathcal{C}^{1+\theta}(X)$ for some $\theta \in(0,1]$ and are hyperbolic, that is, $0<\left|\phi_{j}^{\prime}(x)\right|<1$ on $X$. Then the Hausdorff dimension $\operatorname{dim}_{\mathrm{H}}\left(J_{\Phi}\right)$ is given by Bowen's formula $[\mathbf{B o 2}, \mathbf{R}]$ :

$$
\operatorname{dim}_{\mathrm{H}}\left(J_{\Phi}\right)=s(\Phi) \quad \text { where } \quad P_{\Phi}(s(\Phi))=0 .
$$

Here $P_{\Phi}(t)$ is the pressure function, which can be defined by

$$
P_{\Phi}(t)=\lim _{n \rightarrow \infty} \frac{1}{n} \log \sum_{\omega \in I^{n}}\left\|\phi_{\omega}^{\prime}\right\|^{t}
$$

where $I=\{1, \ldots, k\}, \quad \phi_{\omega}=\phi_{\omega_{1}} \circ \cdots \circ \phi_{\omega_{n}}$, and $\|\cdot\|$ is the supremum norm on $X$.

The dimension formula was extended in $[\mathbf{U}]$ to the parabolic case, where some of the maps have a neutral fixed point. A parabolic IFS is not uniformly contracting, which makes the situation more subtle. It was proved in $[\mathbf{U}]$ 
that the Hausdorff dimension of the limit set is the least zero of the pressure function. In contrast with the hyperbolic case (where the zero is unique), in the parabolic case the pressure function is identically zero for all $t$ larger than the least zero.

We study the case when the "pieces" of the limit set are allowed to overlap. One-dimensional IFS with overlaps arise naturally in the study of higherdimensional dynamical systems $[\mathbf{P r U}, \mathbf{B U}, \mathbf{S i 1}, \mathbf{S i 2}, \mathbf{S S o}]$ (in this sentence the "dimension" refers to the phase space rather than to the limit set). Moreover, IFS with overlaps occur in some problems on random matrix products, random continued fractions, and in prediction theory, see $[\mathbf{P i}, \mathbf{L y}$, LL].

There are many open problems related to IFS with overlaps, which are notoriously difficult even when the maps are linear, see [PSo3]. The dimension of the limit set may be strictly less than the least zero of the pressure function, for instance, if $\phi_{\omega} \equiv \phi_{\tau}$ for two distinct words $\omega$ and $\tau$. Since it is often hard to analyze an individual system, one can try to investigate a "typical" (in the sense of Lebesgue measure) IFS in a parameterized family. This method was first used by Falconer [F1] who considered families of linear contractions with a linear dependence on parameter. Further work in this direction was done in $[\mathbf{P o S}$, So1, PSo2, So2, SSo]. An important role in these papers was played by a certain "transversality condition", which controls the way the IFS depends on parameters.

In this paper this approach is extended to a class of non-linear IFS. Our main result (Theorem 6.1) states that if a parameterized family $\left\{\Phi^{\mathbf{t}}\right\}$ of parabolic IFS satisfies the transversality condition, then for Lebesgue-a.e. parameter $\mathbf{t}$ the Hausdorff dimension of the limit set is given by

$$
\operatorname{dim}_{\mathrm{H}}\left(J_{\Phi^{\mathbf{t}}}\right)=\min \{1, s(\mathbf{t})\} \quad \text { where } \quad s(\mathbf{t}):=\min \left\{s: P_{\Phi^{\mathbf{t}}}(s)=0\right\} .
$$

Moreover, the limit set $J_{\Phi^{\mathrm{t}}}$ has positive Lebesgue measure for a.e. $\mathbf{t}$ such that $s(\mathbf{t})>1$. If a slightly stronger version of transversality is imposed, then the local dimension of the exceptional set in the first statement can be estimated above by $s(\mathbf{t})+(d-1)$, where $d$ is the number of independent parameters involved.

We illustrate our results by the following example (see Corollaries 7.4 and 7.5). Let $\phi(x)=\frac{x}{1+x}$. This function is parabolic on $[0,1]$. Let $A=$ $\left\{a_{1}, \ldots, a_{k}\right\}$, with $k \geq 3$, and consider the family of IFS on $[0,1]$

$$
\begin{aligned}
& \Phi_{A}=\left\{\phi\left(x+a_{j}\right)\right\}_{j=1}^{k} \\
& \quad \text { for } A \in U:=\left\{A \in \mathbb{R}^{k}: a_{k}=0, a_{j}>0, j=1, \ldots, k-1\right\} .
\end{aligned}
$$

Denote by $J_{A}$ the limit set of the IFS $\Phi_{A}$ and let $s(A)=\min \{t>0$ : $\left.P_{\Phi_{A}}(t)=0\right\}$. We will prove that $s: U \rightarrow \mathbb{R}$ is a continuous function, so $U_{<1}=\{A \in U: s(A)<1\}$ and $U_{>1}=\{A \in U: s(A)>1\}$ are open sets. 
Proposition 1.1.

(i) For Lebesgue-a.e. $A \in U$,

$$
\operatorname{dim}_{\mathrm{H}}\left(J_{A}\right)=\min \{s(A), 1\} .
$$

(ii) For any subset $G \subset U_{<1}$ we have

$$
\operatorname{dim}_{\mathrm{H}}\left\{A \in G: \operatorname{dim}_{\mathrm{H}}\left(J_{A}\right)<s(A)\right\} \leq \sup _{G} s(A)+(k-2) .
$$

(iii) For Lebesgue-a.e. $A \in U_{>1}$ the set $J_{A}$ has positive Lebesgue measure.

(iv) Similar results hold for the one-parameter family $\Phi_{A}$ where $A=\{a, 2,0\}$ and $a \in(0,2)$.

Remarks. 1. We assumed that $k \geq 3$ since for $k=2$ either the limit set is an interval, or the IFS has no overlaps, so the result is true for all parameters by $[\mathbf{U}]$.

2. Proposition 1.1(iii) concerning the positive measure of the limit set reflects a phenomenon which cannot occur in the non-overlapping case. It is an open problem whether such limit sets can be "fat" Cantor sets or they necessarily contain intervals.

3. The limit set $J_{A}$ can be described as the set of continued fractions of the form

$$
y=\left[1, Y_{1}, 1, Y_{2}, 1, Y_{3}, \ldots\right]=\frac{1}{1+\frac{1}{Y_{1}+\frac{1}{1+\ldots}}}
$$

where $Y_{i} \in A$. The dimension of sets arising by some restriction in their expansions (continued fractions, $\lambda$-expansions, etc.) was studied by many authors. IFS with overlaps arise when the expansion for some numbers is non-unique. The family of linear IFS $\{\lambda x, \lambda x+1, \lambda x+3\}$, with $\lambda \in\left(\frac{1}{4}, \frac{2}{5}\right)$, investigated in [KSS, PoS, So1], was an important "testing ground" in the study of IFS with overlaps. The family $\{\phi(x), \phi(x+\alpha), \phi(x+2)\}$, with $\alpha \in(0,2)$, that we consider in Proposition 1.1(iv) is a non-linear parabolic analog.

4. A related problem is to analyze invariant (stationary) measures on the limit set of an IFS. The fundamental question is whether this measure is singular or absolutely continuous. This is interesting already for $k=2$, when the limit set is an interval. R. Lyons $[\mathbf{L y}]$ investigated a family of such measures for the IFS $\Phi_{A}$ with $A=\{\alpha, 0\}$. He showed singularity for a certain interval of parameters and asked if the measure is absolutely continuous for small $\alpha$. In [SSU2], using some of the techniques developed in this paper, we establish that the invariant measure is indeed absolutely continuous for a.e. $\alpha$ in some interval.

Here is a brief outline of the contents of the paper.

Section 2 contains preliminaries concerning infinite hyperbolic IFS, including properties of the pressure function. 
Section 3 deals with families of infinite hyperbolic IFS depending on parameters. Its main result, which is of independent interest, is Theorem 3.1, which computes the Hausdorff dimension and Lebesgue measure of the limit set of a.e. infinite hyperbolic IFS with overlaps from a family satisfying a transversality condition.

The exceptional set of parameters, associated with Theorem 3.1, is analyzed in Section 4 where we estimate its local dimension from above.

In Section 5 we consider a single parabolic IFS. Following the approach of [MU2, MU3], we reduce the parabolic IFS to an infinite hyperbolic IFS. The limit sets of the parabolic and infinite hyperbolic systems differ in a countable set, so they have the same dimension. We prove that the unique zero of the pressure for the infinite hyperbolic IFS coincides with the least zero of the pressure for the parabolic IFS, even though the pressure functions for these systems differ (see Proposition 5.10(ii)).

In Section 6 we study families of parabolic IFS. Our main result, Theorem 6.1, computes the Hausdorff dimension and Lebesgue measure of the limit set of a.e. parabolic IFS with overlaps in a family satisfying a transversality condition. Moreover, applying the results of Section 4 we estimate the local dimension of the exceptional set, in the spirit of Proposition 1.1(ii).

Section 7 is devoted to examples. We consider two general classes of examples. The most difficult part in applying Theorem 6.1 is checking the transversality condition. In Propositions 7.1 and 7.2 we obtain effective sufficient conditions for transversality. We conclude with specific examples arising from continued fractions.

Notation. We write $B_{\delta}\left(\mathbf{t}_{0}\right)$ for the open ball of radius $\delta$ centered at $\mathbf{t}_{0}$ and $\mathcal{L}_{d}$ for the Lebesgue measure in $\mathbb{R}^{d}$. If $\mu$ is a measure we often write $\mu A$ without parentheses. The symbol $\preceq$ means that the inequality holds up to an absolute multiplicative constant, and $\asymp$ means that both $\preceq$ and $\succeq$ are true. $\operatorname{Int}(X)$ denotes the interior of a set $X$.

\section{Preliminaries.}

Let $X \subset \mathbb{R}$ be a closed interval. We consider a collection $\Psi=\left\{\psi_{i}\right\}_{i \in I}$ of continuous self-maps of $X$, where the set $I$ may be finite or countable. We set $I^{*}:=\bigcup_{n \geq 1} I^{n}$ and denote by $I^{\infty}$ the set of all infinite sequences of elements of $I$. If $\omega \in I^{*}$, then by $|\omega|$ we denote the length of $\omega$. If $\omega \in I^{*} \cup I^{\infty}$ and $|\omega|>n$ then $\left.\omega\right|_{n}=\omega_{1} \omega_{2} \cdots \omega_{n}$ is the word consisting of the first $n$ letters of $\omega$ and $\sigma^{n} \omega=\omega_{n+1} \omega_{n+2} \cdots \omega_{|\omega|}$. The shift map $\sigma: I^{\infty} \rightarrow I^{\infty}$ sends an element $\left\{\omega_{k}\right\}_{k=1}^{\infty}$ to the element $\left\{\omega_{k+1}\right\}_{k=1}^{\infty}$. If $\omega \in I^{n}$, then by $\psi_{\omega}: X \rightarrow X$ we denote the composition $\psi_{\omega_{1}} \circ \psi_{\omega_{2}} \circ \cdots \circ \psi_{\omega_{n}}$. Notice that given $\omega \in I^{\infty}$, the sequence of compact sets $\left\{\psi_{\left.\omega\right|_{n}}(X)\right\}_{n=1}^{\infty}$ is descending and therefore $\bigcap_{n \geq 1} \psi_{\left.\omega\right|_{n}}(X) \neq \emptyset$. If for every $\omega \in I^{\infty}$ this intersection is a 
singleton, the collection $\Psi$ is said to be a topological IFS. We can then define the map $\pi_{\Psi}: I^{\infty} \rightarrow X$ by setting

$$
\left\{\pi_{\Psi}(\omega)\right\}=\bigcap_{n \geq 1} \psi_{\left.\omega\right|_{n}}(X) .
$$

We call this map the natural projection induced by the topological IFS $\Psi$, and its range, the set $J_{\Psi}=\pi_{\Psi}\left(I^{\infty}\right)$, is called the limit set of $\Psi$. Thus, $\lim _{n \rightarrow \infty} \operatorname{diam}\left(\psi_{\left.\omega\right|_{n}}(X)\right)=0$ and therefore,

$$
\pi_{\Psi}(\omega)=\lim _{n \rightarrow \infty} \psi_{\left.\omega\right|_{n}}(x)
$$

for every $x \in X$. We also have the following useful identity:

$$
\pi_{\Psi}(\omega)=\psi_{\left.\omega\right|_{n}}\left(\pi_{\Psi}\left(\sigma^{n} \omega\right)\right) \quad \text { for any } n \geq 1 .
$$

The limit set satisfies $J_{\Psi}=\bigcup_{i \in I} \psi_{i}\left(J_{\Psi}\right)$ but it need not be compact when $I$ is infinite [MU1]. We call $\Psi$ a smooth IFS if the following condition is satisfied.

Smoothness: There exists $\theta \in(0,1]$ such that

$$
\psi_{i} \in \mathcal{C}^{1+\theta}(X \rightarrow \operatorname{Int}(X)), \quad \text { and } \psi_{i}^{\prime}(x) \neq 0 \text { for all } x \in X \text { and } i \in I \text {. }
$$

Given $t \geq 0$ we define the pressure function $P_{\Psi}(t)$ by the formula

$$
P_{\Psi}(t)=\lim _{n \rightarrow \infty} \frac{1}{n} \log Z_{n}(\Psi, t)
$$

where $Z_{n}(\Psi, t)=\sum_{|\omega|=n}\left\|\psi_{\omega}^{\prime}\right\|^{t}$ and $\|\cdot\|$ denotes the supremum norm on $X$. Observe that the limit in (2.3) really exists since the sequence $n \mapsto$ $\log Z_{n}(\Psi, t)$ is subadditive. Comparing this with the usual definition of topological pressure (see e.g., $[\mathbf{B o 1}]$ ) we see that $P_{\Psi}(t)$ equals the pressure of the shift map $\sigma$ on $I^{\infty}$ with the potential

$$
\omega \mapsto t \log \left|\psi_{\omega_{1}}^{\prime}(\pi(\sigma \omega))\right| .
$$

We call a smooth IFS hyperbolic if the following conditions are satisfied: Hyperbolicity: For some $\gamma \in(0,1)$,

$$
\left\|\psi_{i}^{\prime}\right\| \leq \gamma<1, \quad \text { for all } i \in I
$$

(any map satisfying this property will be called hyperbolic), and

Bounded Distortion Property: There exists $K>1$ such that for all $n \in \mathbb{N}$ and $\omega \in I^{n}$,

$$
K^{-1} \leq \frac{\left|\psi_{\omega}(x)\right|}{\left|\psi_{\omega}(y)\right|} \leq K \quad \text { for all } x, y \in X .
$$

Such $\Psi$ is a one-dimensional example of conformal IFS, introduced (for infinite $I$ ) and explored in [MU1], where also the open set condition was 
assumed. It is well-known that (2.5) follows from (2.2) and (2.4) when $I$ is finite, see e.g., [B1] (see [MU1] for more general sufficient conditions).

From now on throughout the Sections 2-4, unless otherwise stated, we assume that the smooth IFS $\Psi$ is hyperbolic. Let

$$
\Theta(\Psi)=\inf \left\{t \geq 0: P_{\Psi}(t)<\infty\right\} .
$$

The behavior of the pressure function is described in the following lemma.

Lemma 2.1. The function $t \mapsto P_{\Psi}(t)$, for $t \in(\Theta(\Psi), \infty)$, is finite, strictly decreasing and continuous, and $\lim _{t \rightarrow \infty} P_{\Psi}(t)=-\infty$.

Proof. The first statement is immediate from the definition of the number $\Theta=\Theta(\Psi)$. Now, given $t>\Theta$ and $s>0$, we have by (2.4) for all $n \geq 1$ :

$$
Z_{n}(\Psi, t+s)=\sum_{|\omega|=n}\left\|\left(\psi_{\omega}\right)^{\prime}\right\|^{t+s} \leq \sum_{|\omega|=n}\left\|\left(\psi_{\omega}\right)^{\prime}\right\|^{t} \gamma^{n s},
$$

and therefore, $P_{\Psi}(t+s) \leq s \log \gamma+P_{\Psi}(t)<P_{\Psi}(t)$. Thus, the function $t \mapsto P_{\Psi}(t)$ is strictly decreasing on $t \in(\Theta, \infty)$. Now, an application of Hölder's inequality shows that each function $t \mapsto Z_{n}(\Psi, t)$ is $\log$ convex. Therefore the function $t \mapsto P_{\Psi}(t), t \in(\Theta, \infty)$, is convex and, consequently, continuous. By the definition of $\Theta$ we have $P_{\Psi}(\Theta+1)<\infty$. Hence, for every $t>0$ and every $n \geq 1$,

$$
\begin{aligned}
Z_{n}(\Psi, \Theta+1+t)=\sum_{|\omega|=n}\left\|\psi_{\omega}^{\prime}\right\|^{\Theta+1+t} & \leq \sum_{|\omega|=n}\left\|\psi_{\omega}^{\prime}\right\|^{1+\Theta} \cdot\left\|\psi_{\omega}^{\prime}\right\|^{t} \\
& \leq \gamma^{t n} \sum_{|\omega|=n}\left\|\psi_{\omega}^{\prime}\right\|^{1+\Theta} .
\end{aligned}
$$

Therefore, $P_{\Psi}(\Theta+1+t) \leq t \log \gamma+P_{\Psi}(1+\Theta)$ and hence $\lim _{s \rightarrow+\infty} P_{\Psi}(s)=$ $-\infty$. The proof is complete.

Definition 2.2. Following [MU1] we call a hyperbolic system $\Psi$ regular if

$$
\exists s(\Psi) \geq \Theta(\Psi): \quad P_{\Psi}(s(\Psi))=0 .
$$

We denote by $\Xi_{X}(K, \gamma, \theta)$ the class of regular hyperbolic IFS on $X$.

In view of Lemma 2.1, if the number $s(\Psi)$ exists, then it is unique. Also, if $\# I<\infty$ then $\Psi$ is regular since then $\Theta(\Psi)=0$ and $P_{\Psi}(0)=\log (\# I)>0$. The following lemma shows that $s(\Psi)$ is always an upper bound for the Hausdorff dimension of the limit set. The argument is well-known but we include it for completeness. We write $\mathcal{H}^{\alpha}(A)=\lim _{\delta \rightarrow 0} \mathcal{H}_{\delta}^{\alpha}(A)$ for the $\alpha$ dimensional Hausdorff measure of a set $A$.

Lemma 2.3. If $\Psi \in \Xi_{X}(K, \gamma, \theta)$, then $\operatorname{dim}_{\mathrm{H}}\left(J_{\Psi}\right) \leq s(\Psi)$. 
Proof. Fix $\epsilon, \delta>0$ and take $n_{1}$ so large that $\gamma^{n_{1}} \leq \delta$. By the definition of $s(\Psi)$ and Lemma 2.1 there exists $n_{2} \geq n_{1}$ and $\eta>0$ such that for all $n \geq n_{2}$,

$$
\frac{1}{n} \log \left(\sum_{|\omega|=n}\left\|\psi_{\omega}^{\prime}\right\|^{s(\Psi)+\epsilon}\right) \leq-\eta .
$$

Hence, for all $n \geq n_{2}$,

$$
\begin{aligned}
\mathcal{H}_{\delta}^{s(\Psi)+\epsilon}\left(J_{\Psi}\right) & \leq \sum_{|\omega|=n} \operatorname{diam}\left(\psi_{\omega}(X)\right)^{s(\Psi)+\epsilon} \\
& \leq \operatorname{diam}(X)^{s(\Psi)+\epsilon} \sum_{|\omega|=n}\left\|\psi_{\omega}^{\prime}\right\|^{s(\Psi)+\epsilon} \\
& \leq \operatorname{diam}(X)^{s(\Psi)+\epsilon} e^{-\eta n},
\end{aligned}
$$

and, consequently, $\mathcal{H}_{\delta}^{s(\Psi)+\epsilon}\left(J_{\Psi}\right)=0$. Thus, $\mathcal{H}^{s(\Psi)+\epsilon}\left(J_{\Psi}\right)=0$, and letting $\epsilon \searrow 0$ we conclude that $\operatorname{dim}_{\mathrm{H}}\left(J_{\Psi}\right) \leq s(\Psi)$.

Given an IFS $\Psi=\left\{\psi_{i}: i \in I\right\}$ and $F \subset I$ let $\Psi_{F}=\left\{\psi_{i}: i \in F\right\}$. Denote $\operatorname{Fin}(I)=\{F \subset I: \# F<\infty\}$. We are going to show that $s(\Psi)$ is the supremum of $s\left(\Psi_{F}\right)$ over $F \in \operatorname{Fin}(I)$. Along the way we obtain an estimate on the speed of convergence in (2.3), which will be useful later.

Lemma 2.4. Let $\Psi \in \Xi_{X}(K, \gamma, \theta)$. Then

(i) for every $t>0$ and every $n \geq 1$,

$$
P_{\Psi}(t) \leq \frac{1}{n} \log Z_{n}(\Psi, t) \leq P_{\Psi}(t)+\frac{t \log K}{n} .
$$

(ii) $s(\Psi)=\sup \left\{s\left(\Psi_{F}\right): F \in \operatorname{Fin}(I)\right\}$.

Proof. (i) The left-hand side inequality is immediate from the subadditivity of the sequence $n \mapsto \log Z_{n}(\Psi, t)$. In order to prove the right-hand side inequality, fix $n \geq 1$ and consider an arbitrary integer $q \geq 1$. Then

$$
\begin{aligned}
\frac{1}{q n} \log Z_{q n}(\Psi, t) & =\frac{1}{q n} \log \sum_{\omega \in I^{q n}}\left\|\psi_{\omega}^{\prime}\right\|^{t} \\
& \geq \frac{1}{q n} \log \left(K^{-q t} \sum_{\omega \in\left(I^{n}\right)^{q}} \prod_{i=1}^{q}\left\|\psi_{\tau_{i}}^{\prime}\right\|^{t}\right) \\
& =\frac{-t \log K}{n}+\frac{1}{q n} \log \left(\sum_{\tau \in I^{n}}\left\|\psi_{\tau}^{\prime}\right\|^{t}\right)^{q} \\
& =\frac{-t \log K}{n}+\frac{1}{n} \log Z_{n}(\Psi, t),
\end{aligned}
$$


where in the second displayed line we used (2.5) and $\omega=\tau_{1} \ldots \tau_{q}$, with $\tau_{i} \in I^{n}$. Letting $q \rightarrow \infty$ we obtain the right-hand side inequality of (2.8).

(ii) Since for every $t \geq 0$ and every $F \subset I$ we have $P_{\Psi_{F}}(t) \leq P_{\Psi}(t)$, the inequality $s(\Psi) \geq \sup \left\{s\left(\Psi_{F}\right): F \in F \operatorname{Fin}(I)\right\}$ is obvious. The opposite inequality will be deduced from (i). Fix an arbitrary $\Theta(\Psi)<t<s(\Psi)$. Then $0<P_{\Psi}(t)<\infty$ and there exists $n \in \mathbb{N}$ so large that $P_{\Psi}(t)>\frac{2 t \log K}{n}$. Fix such an $n$. Clearly, $Z_{n}(\Psi, t)=\sup \left\{Z_{n}\left(\Psi_{F}\right): F \in F i n(I)\right\}$, hence we can find $F \in \operatorname{Fin}(I)$ satisfying

$$
\frac{\log Z_{n}\left(\Psi_{F}, t\right)}{n} \geq \frac{\log Z_{n}(\Psi, t)}{n}-\frac{t \log K}{n} \geq P_{\Psi}(t)-\frac{t \log K}{n}>\frac{t \log K}{n} .
$$

But now, applying (2.8) to $\Psi_{F} \in \Xi_{X}(K, \gamma, \theta)$ we obtain

$$
P_{\Psi_{F}}(t) \geq \frac{\log Z_{n}\left(\Psi_{F}, t\right)}{n}-\frac{t \log K}{n}>0
$$

which implies that $t \leq s\left(\Psi_{F}\right)$. Thus, $s(\Psi) \leq \sup \left\{s\left(\Psi_{G}\right): G \in \operatorname{Fin}(I)\right\}$, and the proof is complete.

\section{Families of hyperbolic IFS.}

Let $X \subset \mathbb{R}$ be a compact interval and $U \subset \mathbb{R}^{d}$ an open set. Here we consider families of hyperbolic IFS $\Psi^{\mathbf{t}} \in \Xi_{X}(K, \gamma, \theta)$ depending on a parameter $\mathbf{t} \in U$. By $J_{\mathbf{t}}$ we denote the limit set of $\Psi^{\mathbf{t}}$ and by $\pi_{\mathbf{t}}=\pi_{\Psi^{\mathrm{t}}}: I^{\infty} \rightarrow J_{\mathbf{t}}$ we denote the natural projection introduced in Section 2. We need two conditions concerned with the dependence of the IFS on $\mathbf{t}$.

Distortion Continuity: For any $\eta>0$ there exists $\delta>0$ such that

$$
\mathbf{t}_{1}, \mathbf{t}_{2} \in U,\left\|\mathbf{t}_{1}-\mathbf{t}_{2}\right\| \leq \delta \quad \Longrightarrow \quad \forall \omega \in I^{*}, \quad e^{-|\omega| \eta} \leq \frac{\left\|\left(\psi_{\omega}^{\mathbf{t}_{1}}\right)^{\prime}\right\|}{\left\|\left(\psi_{\omega}^{\mathbf{t}_{2}}\right)^{\prime}\right\|} \leq e^{|\omega| \eta} .
$$

Transversality Condition: For any $\omega$ and $\tau$ in $I^{\infty}$ with $\omega_{1} \neq \tau_{1}$, there exists a constant $C_{1}=C_{1}\left(\omega_{1}, \tau_{1}\right)$ such that

$$
\mathcal{L}_{d}\left\{\mathbf{t} \in U:\left|\pi_{\mathbf{t}}(\omega)-\pi_{\mathbf{t}}(\tau)\right| \leq r\right\} \leq C_{1} r \quad \text { for all } r>0 .
$$

We emphasize that $C_{1}$ depends only on $\omega_{1}$ and $\tau_{1}$. Thus, $C_{1}$ can be assumed independent of $\omega$ and $\tau$ if $I$ is finite.

Now we can state the main result of this section. We write $s(\mathbf{t})=s\left(\Psi^{\mathbf{t}}\right)$.

Theorem 3.1. Suppose that $\left\{\Psi^{\mathbf{t}}\right\}_{\mathbf{t} \in U}$ is a family of IFS in $\Xi_{X}(K, \gamma, \theta)$ satisfying (3.1) and (3.2). Then the function $\mathbf{t} \mapsto s(\mathbf{t})$ is continuous on $U$ and

(i) $\operatorname{dim}_{\mathrm{H}}\left(J_{\mathbf{t}}\right)=\min \{s(\mathbf{t}), 1\}$ for Lebesgue-a.e. $\mathbf{t} \in U$;

(ii) $\mathcal{L}_{1}\left(J_{\mathbf{t}}\right)>0$ for Lebesgue-a.e. $\mathbf{t} \in U$ such that $s(\mathbf{t})>1$. 
The rest of the section is devoted to the proof of this theorem. We begin with two lemmas which are easy consequences of (3.1) and (3.2).

Lemma 3.2. Given $\epsilon, a>0$ define $\eta=\frac{-\epsilon \log \gamma}{4 a+\epsilon}$ and take $\delta=\delta(\eta)$ coming from (3.1) ascribed to $\eta$. Then for all $\omega \in I^{*}$,

$$
\left\|\mathbf{t}_{0}-\mathbf{t}\right\|<\delta \quad \Longrightarrow \quad\left\|\left(\psi_{\omega}^{\mathbf{t}_{0}}\right)^{\prime}\right\|^{a+\frac{\epsilon}{4}} \leq\left\|\left(\psi_{\omega}^{\mathbf{t}}\right)^{\prime}\right\|^{a} .
$$

Proof. By (3.1) we have

$$
\begin{aligned}
\left\|\left(\psi_{\omega}^{\mathbf{t}_{0}}\right)^{\prime}\right\|^{a+\frac{\epsilon}{4}} & \leq e^{|\omega| \eta\left(a+\frac{\epsilon}{4}\right)} \cdot\left\|\left(\psi_{\omega}^{\mathbf{t}}\right)^{\prime}\right\|^{a+\frac{\epsilon}{4}} \\
& \leq e^{|\omega| \eta\left(a+\frac{\epsilon}{4}\right)} \cdot \gamma^{|\omega| \frac{\epsilon}{4}} \cdot\left\|\left(\psi_{\omega}^{\mathbf{t}}\right)^{\prime}\right\|^{a} \\
& =e^{|\omega|\left(\eta\left(a+\frac{\epsilon}{4}\right)+\frac{\epsilon}{4} \log \gamma\right)} \cdot\left\|\left(\psi_{\omega}^{\mathbf{t}}\right)^{\prime}\right\|^{a}=\left\|\left(\psi_{\omega}^{\mathbf{t}}\right)^{\prime}\right\|^{a} .
\end{aligned}
$$

The proof is complete.

Lemma 3.3. Suppose that the family $\Psi^{\mathbf{t}}$ satisfies (3.2). Then for every $0<$ $\alpha<1$ and for all $\omega, \tau \in I^{\infty}$ with $\omega_{1} \neq \tau_{1}$, there exists $C_{2}=C_{2}\left(\alpha, \omega_{1}, \tau_{1}\right)>0$ such that

$$
\int_{U} \frac{d \mathbf{t}}{\left|\pi_{\mathbf{t}}(\omega)-\pi_{\mathbf{t}}(\tau)\right|^{\alpha}} \leq C_{2}
$$

Proof. In view of (3.2), we can estimate as follows:

$$
\begin{aligned}
\int_{U} \frac{d \mathbf{t}}{\left|\pi_{\mathbf{t}}(\omega)-\pi_{\mathbf{t}}(\tau)\right|^{\alpha}} & =\int_{0}^{\infty} \mathcal{L}_{d}\left\{\mathbf{t} \in U: \frac{1}{\left|\pi_{\mathbf{t}}(\omega)-\pi_{\mathbf{t}}(\tau)\right|^{\alpha}} \geq x\right\} d x \\
& =\int_{0}^{\infty} \mathcal{L}_{d}\left\{\mathbf{t} \in U:\left|\pi_{\mathbf{t}}(\omega)-\pi_{\mathbf{t}}(\tau)\right| \leq r\right\} r^{-\alpha-1} d r \\
& =\int_{0}^{|X|} \mathcal{L}_{d}\left\{\mathbf{t} \in U:\left|\pi_{\mathbf{t}}(\omega)-\pi_{\mathbf{t}}(\tau)\right| \leq r\right\} r^{-\alpha-1} d r+ \\
& +\int_{|X|}^{\infty} \mathcal{L}_{d}\left\{\mathbf{t} \in U:\left|\pi_{\mathbf{t}}(\omega)-\pi_{\mathbf{t}}(\tau)\right| \leq r\right\} r^{-\alpha-1} d r \\
& \leq C_{1}\left(\omega_{1}, \tau_{1}\right)(1-\alpha)^{-1}|X|^{1-\alpha}+\mathcal{L}_{d}(U) \alpha^{-1}|X|^{-\alpha}
\end{aligned}
$$

and the lemma is proved.

The next lemma is proved following the scheme of [SSo, Lemma 4.1(ii)]; it implies the continuity statement in Theorem 3.1.

Lemma 3.4. If the family $\Psi^{\mathbf{t}} \in \Xi_{X}(K, \gamma, \theta)$, with $\mathbf{t} \in U$, satisfies (3.1), then the function $\mathbf{t} \mapsto s(\mathbf{t})$ is continuous on $U$. 
Proof. Fix an arbitrary $\Phi \in \Xi_{X}(K, \gamma, \theta)$. Then for every $t>\Theta(\Phi)$ and $u \geq 0$ we have

$$
\begin{aligned}
& P_{\Phi}(t+u)-P_{\Phi}(t) \\
& =\lim _{n \rightarrow \infty} \frac{1}{n}\left(\log \left(\sum_{|\omega|=n}\left\|\phi_{\omega}^{\prime}\right\|^{t+u}\right)-\log \left(\sum_{|\omega|=n}\left\|\phi_{\omega}^{\prime}\right\|^{t}\right)\right) \\
& \leq \lim _{n \rightarrow \infty} \frac{1}{n}\left(\log \left(\gamma^{u n} \sum_{|\omega|=n}\left\|\phi_{\omega}^{\prime}\right\|^{t}\right)-\log \left(\sum_{|\omega|=n}\left\|\phi_{\omega}^{\prime}\right\|^{t}\right)\right) \\
& =u \log \gamma .
\end{aligned}
$$

Therefore, for all $t>\Theta(\Phi)$ and $u>\Theta(\Phi)-t$,

$$
\left|P_{\Phi}(t+u)-P_{\Phi}(t)\right| \geq|u| \cdot|\log \gamma| \text {. }
$$

Recall that $P_{\Psi^{\mathbf{t}_{i}}}\left(s\left(\mathbf{t}_{i}\right)\right)=0$ by the definition of $s\left(\mathbf{t}_{i}\right)=s\left(\Psi^{\mathbf{t}_{i}}\right)$. Fix $\epsilon>0$, consider $\delta>0$ produced by (3.1) with $\eta=\epsilon$, and suppose that $\left\|\mathbf{t}_{2}-\mathbf{t}_{1}\right\|<\delta$. Then by (2.3) and (3.1),

$$
\begin{aligned}
\left|P_{\Psi^{\mathbf{t}_{1}}}\left(s\left(\mathbf{t}_{2}\right)\right)\right| & =\left|P_{\Psi^{\mathbf{t}_{1}}}\left(s\left(\mathbf{t}_{2}\right)\right)-P_{\Psi^{\mathbf{t}_{2}}}\left(s\left(\mathbf{t}_{2}\right)\right)\right| \\
& =\lim _{n \rightarrow \infty} \frac{1}{n} \log \left|\frac{\sum_{|\omega|=n}\left\|\left(\psi_{\omega}^{\mathbf{t}_{1}}\right)^{\prime}\right\|^{s\left(\mathbf{t}_{2}\right)}}{\sum_{|\omega|=n}\left\|\left(\psi_{\omega}^{\mathbf{t}_{2}}\right)^{\prime}\right\|^{s\left(\mathbf{t}_{2}\right)}}\right| \leq s\left(\mathbf{t}_{2}\right) \epsilon .
\end{aligned}
$$

Therefore, $s\left(\mathbf{t}_{2}\right)>\Theta\left(\Psi^{\mathbf{t}_{1}}\right)$ and in view of (3.3) we have

$$
\begin{aligned}
\left|s\left(\mathbf{t}_{2}\right)-s\left(\mathbf{t}_{1}\right)\right| & \leq \frac{1}{|\log \gamma|}\left|P_{\Psi^{\mathbf{t}_{1}}}\left(s\left(\mathbf{t}_{2}\right)\right)-P_{\Psi^{\mathbf{t}_{1}}}\left(s\left(\mathbf{t}_{1}\right)\right)\right| \\
& =\frac{1}{|\log \gamma|}\left|P_{\Psi^{\mathbf{t}_{1}}}\left(s\left(\mathbf{t}_{2}\right)\right)\right| \leq \frac{s\left(\mathbf{t}_{2}\right) \epsilon}{|\log \gamma|},
\end{aligned}
$$

and the desired statement follows.

Following [SSo], we now prove the main ingredient needed for the proof of Theorem 3.1.

Lemma 3.5. Suppose that the family $\left\{\Psi^{\mathbf{t}}\right\}_{\mathbf{t} \in U}$ satisfies (3.1) and (3.2). Then

(i) for any $\mathbf{t}_{0} \in U$ and any $\epsilon>0$ there exists $\delta>0$ such that

$$
\operatorname{dim}_{\mathrm{H}}\left(J_{\mathbf{t}}\right) \geq \min \left\{s\left(\mathbf{t}_{0}\right), 1\right\}-\epsilon \quad \text { for } \quad \mathcal{L}_{d^{-}} \text {a.e. } \mathbf{t} \in B_{\delta}\left(\mathbf{t}_{0}\right) .
$$

(ii) Suppose that $s\left(\mathbf{t}_{0}\right)>1+\epsilon$ for some $0<\epsilon<1$. Then there exists $\delta>0$ such that

$$
\mathcal{L}_{1}\left(J_{\mathbf{t}}\right)>0 \quad \text { for } \quad \mathcal{L}_{d^{-}} \text {a.e. } \mathbf{t} \in B_{\delta}\left(\mathbf{t}_{0}\right) .
$$


Proof. Let $s=\min \left\{s\left(\mathbf{t}_{0}\right), 1\right\}$. By Lemma 2.4(ii), there exists a finite subset $F$ of $I$ such that $s\left(\Psi_{F}^{\mathbf{t}_{0}}\right)>s\left(\mathbf{t}_{0}\right)-\frac{\epsilon}{2} \geq s-\frac{\epsilon}{2}$. To simplify notation we set

$$
\Psi=\left\{\psi_{i}\right\}_{i \in F}=\Psi_{F}^{\mathbf{t}_{0}} .
$$

Consider the function $f: F^{\infty} \rightarrow \mathbb{R}$ defined by $f(\omega)=\log \left|\psi_{\omega_{1}}^{\prime}\left(\pi_{\Psi}(\sigma \omega)\right)\right|$. It follows from (2.2) that $f$ is Hölder continuous, and (2.1) implies

$$
\sum_{i=0}^{n-1} f\left(\sigma^{i} \omega\right)=\log \left|\psi_{\left.\omega\right|_{n}}^{\prime}\left(\pi_{\Psi}\left(\sigma^{n} \omega\right)\right)\right| \quad \text { for all } \omega \in F^{\infty} .
$$

Since $P_{\Psi}(s(\Psi))=0$, the theory of Gibbs states (see [Bo1], cf. [MU1] for a more general setting) produces a Borel probability shift-invariant measure $\mu$ on $F^{\infty}$ such that for some constant $C_{3} \geq 1$, all $\omega \in F^{\infty}$, and all $n \geq 1$,

$$
\mu\left[\left.\omega\right|_{n}\right] \in\left(C_{3}^{-1}, C_{3}\right)\left|\psi_{\left.\omega\right|_{n}}^{\prime}\left(\pi_{\Psi}\left(\sigma^{n} \omega\right)\right)\right|^{s(\Psi)} .
$$

Here $\left[\left.\omega\right|_{n}\right]$ is the cylinder set of all sequences starting with $\omega_{1} \ldots \omega_{n}$. The measure $\mu$ is called the Gibbs state for the potential $\omega \mapsto s(\Psi) f(\omega)$. Bounded distortion (2.5) implies that there exists a constant $C_{4} \geq 1$ such that for all $\omega \in F^{\infty}$ and all $n \geq 1$,

$$
\mu\left[\left.\omega\right|_{n}\right] \in\left(C_{4}^{-1}, C_{4}\right)\left\|\psi_{\left.\omega\right|_{n}}^{\prime}\right\|^{s(\Psi)} .
$$

Denote the product measure $\mu \times \mu$ by $\mu_{2}$. First we prove part (i) of the lemma. By the potential-theoretic characterization of the Hausdorff dimension (see $[\mathbf{F} 2$, p. 79$]$ ) it is enough to show that

$$
R(\mathbf{t})=\iint_{F^{\infty} \times F^{\infty}} \frac{d \mu_{2}(\omega, \tau)}{\left|\pi_{\mathbf{t}}(\omega)-\pi_{\mathbf{t}}(\tau)\right|^{s-\epsilon}}<\infty
$$

for a.e. $\mathbf{t} \in B_{\delta}\left(\mathbf{t}_{0}\right)$, where $\pi_{\mathbf{t}}=\pi_{\Psi^{\mathbf{t}}}$. Indeed, (3.5) means that the $(s-\epsilon)$ energy of the "push-down" measure $\mu \circ \pi_{\mathbf{t}}^{-1}$, supported on the limit set $J_{\Psi_{F}^{\mathrm{t}}} \subset J_{\mathbf{t}}$, is finite.

Following the scheme of Kaufman $[\mathbf{K}]$ we prove that

$$
\int_{B_{\delta}\left(\mathbf{t}_{0}\right)} R(\mathbf{t}) d \mathbf{t}<\infty
$$

where $\delta=\delta(\eta)$ comes from (3.1) and $\eta=\frac{-\epsilon \log \gamma}{4\left(s(\Psi)-\frac{\epsilon}{2}\right)+\epsilon}$. For $\rho \in F^{n}$ denote

$$
A_{\rho}=\left\{(\omega, \tau) \in F^{\infty} \times F^{\infty}: \omega \wedge \tau=\rho\right\}
$$

where $\omega \wedge \tau$ is the largest common initial segment of $\omega$ and $\tau$. For $(\omega, \tau) \in A_{\rho}$ we have by (2.1), (2.5), and the Mean Value Theorem:

$$
\begin{aligned}
\left|\pi_{\mathbf{t}}(\omega)-\pi_{\mathbf{t}}(\tau)\right| & =\left|\left(\psi_{\rho}^{\mathbf{t}}\right)^{\prime}(c)\right| \cdot\left|\pi_{\mathbf{t}}\left(\sigma^{n} \omega\right)-\pi_{\mathbf{t}}\left(\sigma^{n} \tau\right)\right| \\
& \geq K^{-1}\left\|\left(\psi_{\rho}^{\mathbf{t}}\right)^{\prime}\right\| \cdot\left|\pi_{\mathbf{t}}\left(\sigma^{n} \omega\right)-\pi_{\mathbf{t}}\left(\sigma^{n} \tau\right)\right| .
\end{aligned}
$$


By Lemma 3.2,

$$
\left\|\left(\psi_{\rho}^{\mathbf{t}}\right)^{\prime}\right\|^{s-\epsilon} \geq\left\|\left(\psi_{\rho}^{\mathbf{t}}\right)^{\prime}\right\|^{s(\Psi)-\frac{\epsilon}{2}} \geq\left\|\psi_{\rho}^{\prime}\right\|^{s(\Psi)-\frac{\epsilon}{4}} \text { for } \mathbf{t} \in B_{\delta}\left(\mathbf{t}_{0}\right)
$$

where $\psi_{\rho}=\psi_{\rho}^{\mathbf{t}_{0}}$. Now we can estimate using (3.6) and (3.7):

$$
\begin{aligned}
& \int_{B_{\delta}\left(\mathbf{t}_{0}\right)} R(\mathbf{t}) d \mathbf{t}=\sum_{n \geq 0} \sum_{\rho \in F^{n}} \iint_{A_{\rho}}\left(\int_{B_{\delta}\left(\mathbf{t}_{0}\right)} \frac{d \mathbf{t}}{\left|\pi_{\mathbf{t}}(\omega)-\pi_{\mathbf{t}}(\tau)\right|^{s-\epsilon}}\right) d \mu_{2}(\omega, \tau) \\
& \preceq \sum_{n \geq 0} \sum_{\rho \in F^{n}} \iint_{A_{\rho}}\left[\left\|\psi_{\rho}^{\prime}\right\|^{-s(\Psi)+\frac{\epsilon}{4}} \int_{B_{\delta}\left(\mathbf{t}_{0}\right)} \frac{d \mathbf{t}}{\left|\pi_{\mathbf{t}}\left(\sigma^{n} \omega\right)-\pi_{\mathbf{t}}\left(\sigma^{n} \tau\right)\right|^{s-\epsilon}}\right] d \mu_{2}(\omega, \tau) \\
& \preceq \sum_{n \geq 0} \sum_{\rho \in F^{n}} \iint_{A_{\rho}}\left\|\psi_{\rho}^{\prime}\right\|^{-s(\Psi)+\frac{\epsilon}{4}} d \mu_{2}(\omega, \tau) .
\end{aligned}
$$

In the last inequality we applied Lemma 3.3 which is possible because $\left(\sigma^{n} \omega\right)_{1} \neq\left(\sigma^{n} \tau\right)_{1}$. The multiplicative constant depends on $\left(\sigma^{n} \omega\right)_{1}$ and $\left(\sigma^{n} \tau\right)_{1}$, but since the set $F$ is finite this does not cause a problem. Next, applying (3.4) and (2.4) and denoting by $[\rho]$ the cylinder set of $\rho \in F^{n}$ we obtain:

$$
\begin{aligned}
\int_{B_{\delta}\left(\mathbf{t}_{0}\right)} R(\mathbf{t}) d \mathbf{t} & \preceq \sum_{n \geq 0} \sum_{\rho \in F^{n}} \iint_{A_{\rho}} \frac{\left\|\psi_{\rho}^{\prime}\right\|^{\frac{\epsilon}{4}}}{\mu[\rho]} d \mu_{2}(\omega, \tau) \\
& \preceq \sum_{n \geq 0} \gamma^{\frac{n \epsilon}{4}} \sum_{\rho \in F^{n}} \frac{\mu_{2}\left(A_{\rho}\right)}{\mu[\rho]} \\
& \leq \sum_{n \geq 0} \gamma^{\frac{n \epsilon}{4}} \sum_{\rho \in F^{n}} \frac{(\mu[\rho])^{2}}{\mu[\rho]}=\sum_{n \geq 0} \gamma^{\frac{n \epsilon}{4}}<\infty .
\end{aligned}
$$

This concludes the proof of part (i).

(ii) Let $\eta=\frac{-\epsilon \log \gamma}{4+\epsilon}$ and determine $\delta=\delta(\eta)$ from (3.1). In view of Lemma 2.4, there exists a finite set $F \subset I$ such that $s\left(\Psi_{F}^{\mathbf{t}_{0}}\right) \geq 1+\frac{\epsilon}{2}$. We use the same set-up as in the proof of part (i) and let $\Psi=\Psi_{F}^{\mathbf{t}_{0}}$. Recall that $\mu$ is the Gibbs state for the potential $\omega \mapsto s(\Psi) \log \left|\psi_{\omega_{1}}^{\prime}\left(\pi_{\Psi}(\sigma \omega)\right)\right|$ satisfying (3.4). For every $\mathbf{t} \in B_{\delta}\left(\mathbf{t}_{0}\right)$ consider

$$
\nu_{\mathbf{t}}=\mu \circ \pi_{\mathbf{t}}^{-1},
$$

the push-down measure on the limit set $J_{\Psi_{F}^{\mathbf{t}}} \subset J_{\mathbf{t}}$. It is enough to show that $\nu_{\mathbf{t}}$ is absolutely continuous with respect to the Lebesgue measure $\mathcal{L}_{1}$ for a.e. $\mathbf{t} \in B_{\delta}\left(\mathbf{t}_{0}\right)$. We prove that

$$
\mathcal{I}=\int_{B_{\delta}\left(\mathbf{t}_{0}\right)} \int_{\mathbb{R}} \underline{D}\left(\nu_{\mathbf{t}}, x\right) d \nu_{\mathbf{t}} d \mathbf{t}<\infty
$$


where

$$
\underline{D}\left(\nu_{\mathbf{t}}, x\right)=\liminf _{r \searrow 0} \frac{\nu_{\mathbf{t}}[x-r, x+r]}{2 r}
$$

is the lower density of the measure $\nu_{\mathbf{t}}$ at the point $x$. This will be sufficient since then for a.e. $\mathbf{t} \in B_{\delta}\left(\mathbf{t}_{0}\right)$ we will have $\underline{D}\left(\nu_{\mathbf{t}}, x\right)<\infty$ for $\nu_{\mathbf{t}}$-a.e. $x$ and [M, Lemma 2.12] will imply that $\nu_{\mathbf{t}}$ is absolutely continuous. The argument below follows the scheme of [PSo1]. First we apply Fatou's Lemma to get

$$
\mathcal{I} \leq \liminf _{r \searrow 0} \int_{B_{\delta}\left(\mathbf{t}_{0}\right)} \int_{\mathbb{R}} \frac{\nu_{\mathbf{t}}[x-r, x+r]}{2 r} d \nu_{\mathbf{t}} d \mathbf{t} .
$$

Next we use the definition of $\nu_{\mathbf{t}}$ to change the variable, write $\nu_{\mathbf{t}}[x-r, x+r]$ as an integral of the indicator function, and change the variable once again to obtain

$$
\int_{\mathbb{R}} \nu_{\mathbf{t}}[x-r, x+r] d \nu_{\mathbf{t}}=\iint_{F^{\infty} \times F^{\infty}} \mathbf{1}_{\left\{\omega \in F^{\infty}:\left|\pi_{\mathbf{t}}(\omega)-\pi_{\mathbf{t}}(\tau)\right| \leq r\right\}} d \mu_{2}(\omega, \tau) .
$$

Substituting this into (3.8) and exchanging the order of integration yields

$$
\begin{aligned}
& \mathcal{I} \leq \liminf _{r \searrow 0}(2 r)^{-1} \iint_{F^{\infty} \times F^{\infty}} \mathcal{L}_{d}\left\{\mathbf{t} \in B_{\delta}\left(\mathbf{t}_{0}\right):\right. \\
&\left.\left|\pi_{\mathbf{t}}(\omega)-\pi_{\mathbf{t}}(\tau)\right| \leq r\right\} d \mu_{2}(\omega, \tau) \\
&=\liminf _{r \searrow 0}(2 r)^{-1} \sum_{n \geq 0} \sum_{\rho \in F^{n}} \iint_{A_{\rho}} \mathcal{L}_{d}\left\{\mathbf{t} \in B_{\delta}\left(\mathbf{t}_{0}\right):\right. \\
&\left.\left|\pi_{\mathbf{t}}(\omega)-\pi_{\mathbf{t}}(\tau)\right| \leq r\right\} d \mu_{2}(\omega, \tau) .
\end{aligned}
$$

By (3.6), Lemma 3.2, and (3.2), we have for all $(\omega, \tau) \in A_{\rho}$ :

$$
\begin{aligned}
& \mathcal{L}_{d}\left\{\mathbf{t} \in B_{\delta}\left(\mathbf{t}_{0}\right):\left|\pi_{\mathbf{t}}(\omega)-\pi_{\mathbf{t}}(\tau)\right| \leq r\right\} \\
\preceq & \mathcal{L}_{d}\left\{\mathbf{t} \in B_{\delta}\left(\mathbf{t}_{0}\right):\left|\pi_{\mathbf{t}}\left(\sigma^{n} \omega\right)-\pi_{\mathbf{t}}\left(\sigma^{n} \tau\right)\right| \leq \frac{K r}{\left\|\left(\psi_{\rho}^{\mathbf{t}}\right)^{\prime}\right\|}\right\} \\
\leq & \mathcal{L}_{d}\left\{\mathbf{t} \in B_{\delta}\left(\mathbf{t}_{0}\right):\left|\pi_{\mathbf{t}}\left(\sigma^{n} \omega\right)-\pi_{\mathbf{t}}\left(\sigma^{n} \tau\right)\right| \leq \frac{K r}{\left\|\psi_{\rho}^{\prime}\right\|^{1+\frac{\epsilon}{4}}}\right\} \preceq r\left\|\psi_{\rho}^{\prime}\right\|^{-1-\frac{\epsilon}{4}} .
\end{aligned}
$$

Here we used again that the constant in (3.2) can be made independent of $\omega$ and $\tau$, due to the fact that $F$ is finite. Now we can estimate the integral $\mathcal{I}$ as follows:

$$
\mathcal{I} \preceq \sum_{n \geq 0} \sum_{\rho \in F^{n}} \iint_{A_{\rho}}\left\|\psi_{\rho}^{\prime}\right\|^{-1-\frac{\epsilon}{4}} d \mu_{2}(\omega, \tau) .
$$

By (3.4) and (2.4),

$$
\left\|\psi_{\rho}^{\prime}\right\|^{-1-\frac{\epsilon}{4}} \asymp(\mu[\rho])^{-\frac{1+\epsilon / 4}{s(\Psi)}} \leq(\mu[\rho])^{-\left(1-\frac{\epsilon}{12}\right)} \preceq \gamma^{\frac{n \epsilon}{12}}(\mu[\rho])^{-1},
$$


since $s(\Psi)>1+\frac{\epsilon}{2}$ and $\left(1+\frac{\epsilon}{4}\right) /\left(1+\frac{\epsilon}{2}\right)<1-\frac{\epsilon}{12}$ for $\epsilon<1$. Thus,

$$
\mathcal{I} \preceq \sum_{n \geq 0} \gamma^{\frac{n \epsilon}{12}} \sum_{\rho \in F^{n}} \frac{\mu_{2}\left(A_{\rho}\right)}{\mu[\rho]} \leq \sum_{n \geq 0} \gamma^{\frac{n \epsilon}{12}} \sum_{\rho \in F^{n}} \mu[\rho]=\sum_{n \geq 0} \gamma^{\frac{n \epsilon}{12}}<\infty .
$$

The proof is complete.

Proof of Theorem 3.1. By Lemma 2.3, the function $s(\mathbf{t})=\min \left\{s\left(\Psi^{\mathbf{t}}\right), 1\right\}$ is an upper bound for the Hausdorff dimension of the limit set $J_{\mathbf{t}}$. So we just have to show that

$$
\operatorname{dim}_{\mathrm{H}}\left(J_{\mathbf{t}}\right) \geq s(\mathbf{t})
$$

for a.e. $\mathbf{t} \in U$. Suppose that this is not the case. Then we can find $\epsilon>0$ and $\mathbf{t}_{0}$, a density point of those $\mathbf{t}$ for which

$$
\operatorname{dim}_{\mathrm{H}}\left(J_{\mathbf{t}}\right)<s(\mathbf{t})-\epsilon .
$$

Then there exists $\delta_{0}>0$ such that for each $\delta<\delta_{0}$,

$$
\mathcal{L}_{d}\left\{\mathbf{t} \in B_{\delta}\left(\mathbf{t}_{0}\right): \operatorname{dim}_{\mathrm{H}}\left(J_{\mathbf{t}}\right)<\min \{s(\mathbf{t}), 1\}-\epsilon\right\}>0 .
$$

However, by the continuity of the function $s(\mathbf{t})$ (see Lemma 3.4), if $\delta$ is small enough then $s(\mathbf{t})<s\left(\mathbf{t}_{0}\right)+\frac{\epsilon}{2}$ for all $\mathbf{t} \in B_{\delta}\left(\mathbf{t}_{0}\right)$. Thus, for all $\delta$ sufficiently small we obtain from (3.9) that

$$
\mathcal{L}_{d}\left\{\mathbf{t} \in B_{\delta}\left(\mathbf{t}_{0}\right): \operatorname{dim}_{\mathrm{H}}\left(J_{\mathbf{t}}\right)<\min \left\{s\left(\mathbf{t}_{0}\right), 1\right\}-\frac{\epsilon}{2}\right\}>0 .
$$

This contradicts Lemma 3.5(i) and completes the proof of the first part of Theorem 3.1. The second part follows immediately from Lemma 3.5(ii).

\section{Exceptional parameters.}

In this section, following the scheme of Kaufman $[\mathbf{K}]$, we obtain an estimate from above for the local Hausdorff dimension of the set of exceptional parameters in Theorem 3.1(i). As in Section 3, we assume that $\left\{\Psi^{\mathbf{t}}\right\}_{\mathbf{t} \in U}$ is a family of IFS in $\Xi_{X}(K, \gamma, \theta)$ satisfying (3.1), but we will need the following stronger transversality condition which will be checked for all the examples that we consider. Denote by $N_{r}(F)$ the minimal number of balls of radius $r$ needed to cover the set $F \subset \mathbb{R}^{d}$.

Strong Transversality Condition: For all $\omega$ and $\tau$ in $I^{\infty}$ with $\omega_{1} \neq \tau_{1}$, there exists a constant $C_{1}=C_{1}\left(\omega_{1}, \tau_{1}\right)$ such that for all $r>0$,

$$
N_{r}\left(\left\{\mathbf{t} \in U:\left|\pi_{\mathbf{t}}(\omega)-\pi_{\mathbf{t}}(\tau)\right| \leq r\right\}\right) \leq C_{1} r^{1-d} .
$$

Of course, the strong transversality condition implies the transversality condition (3.2). In the same way as Lemma 3.3 we can prove the following. 
Lemma 4.1. Suppose that the family $\Psi^{\mathrm{t}}$ satisfies the strong transversality condition (4.1). Let $m$ be a Borel probability measure in $\mathbb{R}^{d}$ such that $m\left(B_{r}(x)\right) \leq C r^{u}$ for some $C, u>0$ and all $x \in \mathbb{R}^{d}, r>0$. Then for every $\alpha<u-d+1$ and for all $\omega, \tau \in \mathcal{A}^{\infty}$ with $\omega_{1} \neq \tau_{1}$, there exists $C_{2}=C_{2}\left(\alpha, \omega_{1}, \tau_{1}\right)>0$ such that

$$
\int_{U} \frac{d m(\mathbf{t})}{\left|\pi_{\mathbf{t}}(\omega)-\pi_{\mathbf{t}}(\tau)\right|^{\alpha}} \leq C_{2}
$$

In the sequel any measure with the properties required in Lemma 4.1 will be called a Frostman measure with exponent $u$. Next we prove the analog of Lemma 3.5(i).

Lemma 4.2. Suppose that the family $\left\{\Psi^{\mathbf{t}}\right\}_{\mathbf{t} \in U}$ satisfies (3.1) and (4.1). Then for any $\mathbf{t}_{0} \in U$ and any $\epsilon>0$ there exists $\delta=\delta\left(\mathbf{t}_{0}, \epsilon\right)>0$ such that if $m$ is a Frostman measure on $B_{\delta}\left(\mathbf{t}_{0}\right)$ with exponent $u$, then

$$
\operatorname{dim}_{\mathbf{H}}\left(J_{\mathbf{t}}\right) \geq \min \left\{s\left(\mathbf{t}_{0}\right), u-d+1\right\}-\epsilon
$$

for $m$-a.e. $\mathbf{t} \in B_{\delta}\left(\mathbf{t}_{0}\right)$.

Proof. We let $s=\min \left\{s\left(\mathbf{t}_{0}\right), u-d+1\right\}$ and then repeat the proof of Lemma 3.5(i) almost word by word. The only change is that now we prove that $\int_{B_{\delta}\left(\mathbf{t}_{0}\right)} R(\mathbf{t}) d m(\mathbf{t})<\infty$ using Lemma 4.1 in the place where Lemma 3.3 was used.

Now we can prove the main result of this section.

Theorem 4.3. Suppose that the d-parameter family of IFS $\left\{\Psi^{\mathbf{t}}\right\}_{\mathbf{t} \in U}$ satisfies (3.1) and (4.1). If $G$ is an arbitrary subset of $U$, then for every $\xi>0$ we have

$$
\operatorname{dim}_{\mathrm{H}}\left(\left\{\mathbf{t} \in G: \operatorname{dim}_{\mathrm{H}}\left(J_{t}\right)<\min \{\xi, s(\mathbf{t})\}\right\}\right) \leq \min \left\{\xi, \sup _{G} s(\mathbf{t})\right\}+d-1 .
$$

Proof. Denote $\kappa:=\min \left\{\xi, \sup _{G} s(\mathbf{t})\right\}+d-1$. By the countable stability of the Hausdorff dimension, it is enough to prove that for all $n \in \mathbb{N}$,

$$
\operatorname{dim}_{\mathrm{H}}\left(\left\{\mathbf{t} \in G: \operatorname{dim}_{\mathrm{H}}\left(J_{t}\right)<\min \{\xi, s(\mathbf{t})\}-\frac{1}{n}\right\}\right) \leq \kappa .
$$

Fix $n$ and observe that it suffices to show that for all $\mathbf{t}_{0}$ in $G$ there exists $\delta=\delta\left(\mathbf{t}_{0}\right)$ such that

$$
\operatorname{dim}_{\mathrm{H}}\left(\left\{\mathbf{t} \in B_{\delta}\left(\mathbf{t}_{0}\right): \operatorname{dim}_{\mathrm{H}}\left(J_{t}\right)<\min \{\xi, s(\mathbf{t})\}-\frac{1}{n}\right\}\right) \leq \kappa
$$

(just use that any cover of $G$ contains a countable subcover and again the countable stability of the Hausdorff dimension). To establish our claim, suppose that it is false. Then there exists $\mathbf{t}_{0}$ such that for all $\delta>0$

$$
\operatorname{dim}_{\mathrm{H}}\left(\left\{\mathbf{t} \in B_{\delta}\left(\mathbf{t}_{0}\right): \operatorname{dim}_{\mathrm{H}}\left(J_{t}\right)<\min \{\xi, s(\mathbf{t})\}-\frac{1}{n}\right\}\right)>\kappa .
$$


Choose $\delta>0$ so small that the statement of Lemma 4.2 holds with $\epsilon=\frac{1}{2 n}$ and $\left|s(\mathbf{t})-s\left(\mathbf{t}_{0}\right)\right|<\frac{1}{2 n}$ for all $\mathbf{t} \in B_{\delta}\left(\mathbf{t}_{0}\right)$ (by the continuity of $\left.s(\mathbf{t})\right)$. Then

$$
\begin{aligned}
& \left\{\mathbf{t} \in B_{\delta}\left(\mathbf{t}_{0}\right): \operatorname{dim}_{\mathrm{H}}\left(J_{t}\right)<\min \{\xi, s(\mathbf{t})\}-\frac{1}{n}\right\} \\
\subset & \left\{\mathbf{t} \in B_{\delta}\left(\mathbf{t}_{0}\right): \operatorname{dim}_{\mathrm{H}}\left(J_{t}\right)<\min \left\{\xi, s\left(\mathbf{t}_{0}\right)\right\}-\frac{1}{2 n}\right\}=: E,
\end{aligned}
$$

hence $\operatorname{dim}_{\mathrm{H}}(E)>\kappa$. By Frostman's Lemma (see [M, Th.8.8]), there is a Frostman measure $m$ on the set $E$ with exponent $u=\kappa$. By Lemma 4.2, for $m$-a.e. $\mathbf{t}$ we have

$\operatorname{dim}_{\mathrm{H}}\left(J_{\mathbf{t}}\right) \geq \min \left\{s\left(\mathbf{t}_{0}\right), \kappa-d+1\right\}-\frac{1}{2 n}=\min \left\{s\left(\mathbf{t}_{0}\right), \min \left\{\xi, \sup _{G} s(\mathbf{t})\right\}\right\}-\frac{1}{2 n}$.

This is a contradiction since for all $\mathbf{t} \in E$ we have $\operatorname{dim}_{\mathrm{H}}\left(J_{t}\right)<\min \left\{\xi, s\left(\mathbf{t}_{0}\right)\right\}-$ $\frac{1}{2 n}$ and

$$
\min \left\{\xi, s\left(\mathbf{t}_{0}\right)\right\} \leq \min \left\{s\left(\mathbf{t}_{0}\right), \min \left\{\xi, \sup _{G} s(\mathbf{t})\right\}\right\}
$$

Since the function $\mathbf{t} \mapsto s(\mathbf{t}), \mathbf{t} \in U$, is continuous, as an immediate consequence of Theorem 4.3 we get the following estimate for the local dimension of the exceptional set.

Corollary 4.4. For every $\mathbf{t}_{0} \in U$ we have

$\lim _{r \rightarrow 0} \operatorname{dim}_{\mathrm{H}}\left(\left\{t \in B_{r}\left(\mathbf{t}_{0}\right): \operatorname{dim}_{\mathrm{H}}\left(J_{\mathbf{t}}\right)<\min \{\xi, s(\mathbf{t})\}\right\}\right) \leq \min \left\{\xi, s\left(\mathbf{t}_{0}\right)\right\}+d-1$.

\section{Parabolic IFS.}

Let $X \subset \mathbb{R}$ be a compact interval. We say that a $\mathcal{C}^{1+\theta}$ map $\phi: X \rightarrow X$ is parabolic if the following requirements are fulfilled:

- there is only one point $v \in X$ such that $\phi(v)=v$;

- $\left|\phi^{\prime}(v)\right|=1$ and $0<\left|\phi^{\prime}(x)\right|<1$ for all $x \in X \backslash\{v\}$.

- There exists $L_{1} \geq 1$ and $\beta=\beta(\phi)<\theta /(1-\theta)(=\infty$ if $\theta=1)$ such that

$$
L_{1}^{-1} \leq \liminf _{x \rightarrow v} \frac{|| \phi^{\prime}(x)|-1|}{|x-v|^{\beta}} \leq \limsup _{x \rightarrow v} \frac{|| \phi^{\prime}(x)|-1|}{|x-v|^{\beta}} \leq L_{1} .
$$

At the beginning of this section we state some useful properties of a single parabolic map. They are very similar to [U, Lemmas 2.1-2.3]. First, integrating the partial sums of the series $\sum_{n=1}^{\infty}\left|\left(\phi^{n}\right)^{\prime}(x)\right|$ we get the following. 
Lemma 5.1. For all $x \in \phi(X) \backslash\{v\}$ we have

$$
\frac{|x-v|}{\left|\phi^{-1}(x)-x\right|} \leq \sum_{n=1}^{\infty}\left|\left(\phi^{n}\right)^{\prime}(x)\right| \leq \frac{|\phi(x)-v|}{|x-\phi(x)|}
$$

Sending a sufficiently small neighborhood of $v$ to infinity via the mapping $x \mapsto 1 /(x-v)$, one can easily prove the following two local results.

Lemma 5.2. For every neighborhood $V$ of $v$ there exists $L_{2}(V) \geq 1$ such that for all $x \in X \backslash V$ and all $n \geq 1$,

$$
\frac{1}{L_{2}(V)} \leq\left|\phi^{n}(x)-v\right| \cdot n^{1 / \beta} \leq L_{2}(V) .
$$

Lemma 5.3. For every neighborhood $V$ of $v$ there exists $L_{3}(V) \geq 1$ such that for all $x \in X \backslash V$ and all $n \geq 1$

$$
\frac{1}{L_{3}(V)} \leq\left|\left(\phi^{n}\right)^{\prime}(x)\right| \cdot n^{\frac{\beta+1}{\beta}} \leq L_{3}(V) .
$$

Since $\beta<\theta /(1-\theta)$, the following is immediate from Lemma 5.3.

Corollary 5.4. For every neighborhood $V$ of $v$ there exists $L_{4}(V)<\infty$ such that

$$
\sum_{n=1}^{\infty}\left\|\left(\phi^{n}\right)^{\prime}\right\|_{X \backslash V}^{\theta}<L_{4}(V)
$$

where $\|\cdot\|_{X \backslash V}$ denotes the sup-norm on $X \backslash V$.

Turning now our attention to iterated function systems we recall that a $\mathcal{C}^{1+\theta}$ map $\phi$ is hyperbolic if $0<\left|\phi^{\prime}(x)\right|<1$ for all $x \in X$.

Definition 5.5. Let $\Phi=\left\{\phi_{1}, \ldots, \phi_{k}\right\}$ be a collection of $\mathcal{C}^{1+\theta}$ functions on a closed interval $X \subset \mathbb{R}$ such that $\phi_{k}$ is parabolic with the fixed point $v$ and the other functions are hyperbolic. We write $\Phi \in \Gamma_{X}(\theta)$ if, in addition,

$$
\phi_{i}(X) \subset \operatorname{Int}(X) \backslash\{v\} \quad \text { for all } i \leq k-1 .
$$

Remark. We consider IFS with just one parabolic element. The case of more than one parabolic function can also be handled, but at the cost of additional technical complications.

Let $\mathcal{A}=\{1, \ldots, k\}, \mathcal{A}^{*}:=\bigcup_{n \geq 1} \mathcal{A}^{n}$, and suppose that

$$
\max \left\{\left\|\phi_{i}^{\prime}\right\|: i \leq k-1\right\} \leq \gamma<1 .
$$

Lemma 5.6. An IFS $\Phi \in \Gamma_{X}(\theta)$ is a topological IFS. 
Proof. All we need to show is that the intersections $\bigcap_{n \geq 1} \phi_{\left.\omega\right|_{n}}(X)$ are singletons for all $\omega \in \mathcal{A}^{\infty}$. By (5.2),

$$
\lim _{n \rightarrow \infty} \operatorname{diam}\left(\phi_{\left.\omega\right|_{n}}(X)\right) \rightarrow 0
$$

if the sequence $\omega$ has infinitely many symbols other than $k$. The remaining possibility is $\omega=w k^{\infty}$ for some $w \in \mathcal{A}^{*}$ but then (5.3) is still true since $\bigcap_{n \geq 1} \phi_{k^{n}}(X)=\{v\}$.

Now, following [MU2, MU3], given a parabolic IFS $\Phi \in \Gamma_{X}(\theta)$, consider an associated infinite IFS

$$
\Phi_{*}=\left\{\phi_{k}^{n} \phi_{i}: n \geq 0, i \leq k-1\right\} .
$$

We also write $\Phi_{*}=\left\{\phi_{b}^{*}\right\}_{b \in I}$ where $\phi_{b}^{*}=\phi_{k}^{n} \phi_{i}$ and

$$
I=\{b=(n, i): n \geq 0, i \leq k-1\} .
$$

The following properties of $\Phi_{*}$ are immediate from the definitions.

Corollary 5.7. Let $\Phi \in \Gamma_{X}(\theta)$. Then $\Phi_{*}=\left\{\phi_{b}^{*}\right\}_{b \in I}$ satisfies

(i) $\phi_{b}^{*} \in \mathcal{C}^{1+\theta}(X \rightarrow \operatorname{Int}(X))$ for all $b \in I$;

(ii) $0<\left\|\left(\phi_{b}^{*}\right)^{\prime}\right\| \leq \gamma<1$ for all $b \in I$;

(iii) $J_{\Phi}=J_{\Phi_{*}} \cup\left\{\phi_{w}(v)\right\}_{w \in \mathcal{A}^{*}}$ so $\operatorname{dim}_{\mathrm{H}}\left(J_{\Phi}\right)=\operatorname{dim}_{\mathrm{H}}\left(J_{\Phi_{*}}\right)$.

Thus there is an infinite hyperbolic IFS $\Phi_{*}$ associated with a finite parabolic IFS $\Phi$. This idea essentially goes back to Schweiger's "jump transformation" [Sc]. Our next goal is to show that $\Phi \in \Gamma_{X}(\theta)$ implies $\Phi_{*} \in$ $\Xi_{X}(K, \gamma, \theta)$ for some $K$, see Definition 2.2. To achieve this, two more properties have to be verified: The bounded distortion property (2.5) and regularity (2.7). Bounded distortion properties for parabolic IFS (without overlaps) were investigated in $[\mathbf{U}]$. Here a different version is needed but the approach is similar.

In the next section we study families of parabolic IFS, and it will be very important to know exactly what the various constants depend on. Therefore, we introduce the following notation. Let $\Phi \in \Gamma_{X}(\theta)$. We write

$$
\Phi \in \Gamma_{X}(\theta, V, \gamma, u, M)
$$

if $V$ is a connected open neighborhood of the parabolic point $v$ such that

$$
\begin{gathered}
V \cap \bigcup_{i=1}^{k-1} \phi_{i}(X)=\emptyset, \\
\max \left\{\left\|\left(\phi_{i}\right)^{\prime}\right\|, i \leq k-1\right\} \leq \gamma \in(0,1), \\
\min \left\{\left|\phi_{i}^{\prime}(x)\right|, \quad x \in X, i \leq k\right\} \geq u \in(0,1),
\end{gathered}
$$


and

$$
\left\|\Phi^{\prime}\right\|_{\theta}:=\max _{i \leq k} \sup \left\{\left|\phi_{i}^{\prime}(x)-\phi_{i}^{\prime}(y)\right| \cdot|x-y|^{-\theta}\right\} \leq M .
$$

By Definition 5.5, every $\Phi \in \Gamma_{X}(\theta)$ belongs to some $\Gamma_{X}(\theta, V, \gamma, u, M)$.

The next lemma will also be useful when we consider families of parabolic IFS. Recall that $\|\cdot\|_{X \backslash V}$ denotes the supremum norm on $X \backslash V$.

Lemma 5.8. There exist constants $L_{5}=L_{5}(X, \theta, V, \gamma, u, M)>1$ and $L_{6}=$ $L_{6}(\theta, V, \gamma)>0$ such that for every $\Phi \in \Gamma_{X}(\theta, V, \gamma, u, M)$, all $\omega \in \mathcal{A}^{*}$, and all $x, y \in X \backslash V$,

$$
L_{5}^{-|x-y|^{\theta}} \leq \frac{\left|\phi_{\omega}^{\prime}(y)\right|}{\left|\phi_{\omega}^{\prime}(x)\right|} \leq L_{5}^{|x-y|^{\theta}}
$$

and

$$
\sum_{j=0}^{|\omega|-1}\left\|\phi_{\sigma^{j} \omega}^{\prime}\right\|_{X \backslash V}^{\theta} \leq L_{6} .
$$

Proof. We start with (5.10). Every $\tau \in \mathcal{A}^{*}$ can be written as

$$
\tau=k^{r_{1}} j_{1} k^{r_{2}} j_{2} \ldots k^{r_{l}} j_{l} k^{r_{l+1}}
$$

where $l \geq 0$ and $r_{p} \geq 0, j_{p} \leq k-1$ for $p \leq l$. When $l=0$, Equation (5.11) becomes $\tau=k^{r_{1}}$. One readily estimates using (5.5) and (5.6):

$$
\left\|\phi_{\tau}^{\prime}\right\|_{X \backslash V} \leq \gamma^{l} \prod_{p=1}^{l+1}\left\|\left(\phi_{k}^{r_{p}}\right)^{\prime}\right\|_{X \backslash V} .
$$

Applying this inequality to $\tau=\sigma^{j} \omega$ and summing over $j$ we obtain

$$
\begin{aligned}
& \sum_{j=0}^{|\omega|-1}\left\|\phi_{\sigma^{j} \omega}^{\prime}\right\|_{X \backslash V}^{\theta} \\
& \leq \gamma^{l \theta} \cdot\left(\sum_{j=1}^{r_{1}}\left\|\left(\phi_{k}^{j}\right)^{\prime}\right\|_{X \backslash V}^{\theta}+1\right) \cdot \prod_{p=2}^{l+1} \|\left(\phi_{k}^{\left.r_{p}\right)^{\prime} \|_{X \backslash V}^{\theta}}\right. \\
& \quad+\gamma^{(l-1) \theta} \cdot\left(\sum_{j=1}^{r_{2}}\left\|\left(\phi_{k}^{j}\right)^{\prime}\right\|_{X \backslash V}^{\theta}+1\right) \cdot \prod_{p=3}^{l+1}\left\|\left(\phi_{k}^{r_{p}}\right)^{\prime}\right\|_{X \backslash V}^{\theta} \\
& \quad+\cdots+\gamma^{\theta} \cdot\left(\sum_{j=1}^{r_{l}}\left\|\left(\phi_{k}^{j}\right)^{\prime}\right\|_{X \backslash V}^{\theta}+1\right) \cdot\left\|\left(\phi_{k}^{r_{l+1}}\right)^{\prime}\right\|+\sum_{j=1}^{r_{l+1}}\left\|\left(\phi_{k}^{j}\right)^{\prime}\right\|_{X \backslash V}^{\theta} .
\end{aligned}
$$


Applying Corollary 5.4 for $\phi=\phi_{k}$ and using that $\left\|\left(\phi_{k}^{r}\right)^{\prime}\right\|_{X \backslash V} \leq 1$ for all $r$, we obtain

$$
\sum_{j=0}^{|\omega|-1}\left\|\phi_{\sigma^{j} \omega}^{\prime}\right\|_{X \backslash V}^{\theta} \leq\left(L_{4}(V)+1\right) \sum_{i=0}^{\infty} \gamma^{i \theta}=: L_{6}=L_{6}(\theta, V, \gamma)<\infty,
$$

and (5.10) is proved.

Now we turn to (5.9); clearly, it suffices to prove the right-hand side inequality. Suppose first that $x$ and $y$ belong to the same connected component of $X \backslash V$. Then using the Mean Value Theorem we conclude that for every $0 \leq j \leq|\omega|$ there exists $c_{j} \in X \backslash V$ such that $\left|\phi_{\sigma^{j} \omega}(y)-\phi_{\sigma^{j} \omega}(x)\right|=$ $\left|\phi_{\sigma^{j} \omega}^{\prime}\left(c_{j}\right)\right| \cdot|y-x|$. We have for all $1 \leq i \leq|\omega|$ by $(5.7)$ :

$$
\begin{aligned}
& |\log | \phi_{\omega_{j}}^{\prime}\left(\phi_{\sigma^{j} \omega}(y)\right)|-\log | \phi_{\omega_{j}}^{\prime}\left(\phi_{\sigma^{j} \omega}(x)\right)|| \\
& \leq|| \phi_{\omega_{j}}^{\prime}\left(\phi_{\sigma^{j} \omega}(y)\right)\left|\frac{1}{u}-\right| \phi_{\omega_{j}}^{\prime}\left(\phi_{\sigma^{j} \omega}(x)\right)|| \\
& \leq \frac{\left\|\Phi^{\prime}\right\|_{\theta}}{u}\left|\phi_{\sigma^{j} \omega}(y)-\phi_{\sigma^{j} \omega}(x)\right|^{\theta} \\
& =\frac{\left\|\Phi^{\prime}\right\|_{\theta}}{u}\left|\phi_{\sigma^{j} \omega}^{\prime}\left(c_{j}\right)\right|^{\theta}|y-x|^{\theta} .
\end{aligned}
$$

Since $c_{j} \in X \backslash V$, we obtain

$$
\begin{aligned}
& |\log | \phi_{\omega}^{\prime}(y)|-\log | \phi_{\omega}^{\prime}(x)|| \\
& =\left|\sum_{j=1}^{|\omega|} \log \right| \phi_{\omega_{j}}^{\prime}\left(\phi_{\sigma^{j} \omega}(y)\right)\left|-\sum_{j=1}^{|\omega|} \log \right| \phi_{\omega_{j}}^{\prime}\left(\phi_{\sigma^{j} \omega}(x)\right)|| \\
& \leq \sum_{j=1}^{|\omega|}|\log | \phi_{\omega_{j}}^{\prime}\left(\phi_{\sigma^{j} \omega}(y)\right)|-\log | \phi_{\omega_{j}}^{\prime}\left(\phi_{\sigma^{j} \omega}(x)\right)|| \\
& \leq \sum_{j=1}^{|\omega|} \frac{\left\|\Phi^{\prime}\right\|_{\theta}}{u}\left|\phi_{\sigma^{j} \omega}^{\prime}\left(c_{j}\right)\right|^{\theta}|y-x|^{\theta} \\
& \leq \frac{\left\|\Phi^{\prime}\right\|_{\theta}}{u} L_{6}|y-x|^{\theta} \leq \frac{M}{u} L_{6}|y-x|^{\theta} .
\end{aligned}
$$

In the last displayed line we used (5.10) and (5.8). This completes the proof of (5.9) when $x$ and $y$ are in the same connected component of $X \backslash V$. Now suppose that they are in different components. Since then $|y-x| \geq \operatorname{diam}(V)$, it suffices to show the existence of a constant $L_{7}=L_{7}(X, \theta, V, \gamma, u, M) \geq 1$ such that $\left|\phi_{\omega}^{\prime}(y)\right| \leq L_{7} \cdot\left|\phi_{\omega}^{\prime}(x)\right|$ for all $\omega \in \mathcal{A}^{*}$. To this end, suppose $\omega=\tau k^{n}$ where $n \geq 0$ and $\tau_{|\tau|} \neq k$. Let $|\tau|=l$. Observe that the points $\phi_{\tau_{l} k^{n}}(x)$ and 
$\phi_{\tau_{l} k^{n}}(y)$ belong to $\phi_{\tau_{l}}(X)$ and hence are in the same connected component of $X \backslash V$. Thus, in view of Lemma 5.3 and (5.12) we get

$$
\begin{aligned}
\frac{\left|\phi_{\omega}^{\prime}(y)\right|}{\left|\phi_{\omega}^{\prime}(x)\right|} & =\frac{\left|\phi_{\left.\tau\right|_{l-1}}^{\prime}\left(\phi_{\tau_{l} k^{n}}(y)\right)\right| \cdot\left|\phi_{\tau_{l}}^{\prime}\left(\phi_{k^{n}}(y)\right)\right| \cdot\left|\phi_{k^{n}}^{\prime}(y)\right|}{\left|\phi_{\tau_{l-1}}^{\prime}\left(\phi_{\tau_{l} k^{n}}(x)\right)\right| \cdot\left|\phi_{\tau_{l}}^{\prime}\left(\phi_{k^{n}}(x)\right)\right| \cdot\left|\phi_{k^{n}}^{\prime}(x)\right|} \\
& \leq \exp \left(\frac{M}{u} L_{6}|y-x|^{\theta}\right) \frac{\gamma}{u} L_{3}(V)^{2},
\end{aligned}
$$

and it remains to note that $|y-x| \leq \operatorname{diam}(X)$. The only possibility left is $\omega=k^{n}$ but then $\left|\phi_{k^{n}}^{\prime}(y)\right| \leq L_{3}(V)^{\overline{2}}\left|\phi_{k^{n}}^{\prime}(x)\right|$ by Lemma 5.3. The proof is complete.

Corollary 5.9. There exists a constant $K_{1}=K_{1}(X, \theta, V, \gamma, u, M)>1$ such that for every $\Phi \in \Gamma_{X}(\theta, V, \gamma, u, M)$, the associated infinite IFS $\Phi_{*}$ satisfies (2.5) with $K=K_{1}$. More precisely, for all $\tau \in I^{*}$ and all $x, y \in X$,

$$
K_{1}^{-|y-x|^{\theta}} \leq \frac{\left|\left(\phi_{\tau}^{*}\right)^{\prime}(y)\right|}{\left|\left(\phi_{\tau}^{*}\right)^{\prime}(x)\right|} \leq K_{1}^{|y-x|^{\theta}}
$$

Proof. It is enough to prove only the right-hand side of this formula. By the definition of the system $\Phi_{*}$ we can write $\phi_{\tau}^{*}=\phi_{\omega} \phi_{i}$, where $\omega \in \mathcal{A}^{*}$ and $i \in\{1,2, \ldots, k-1\}$. Then, applying (5.9) and (5.7) we can estimate as follows:

$$
\begin{aligned}
\frac{\left|\left(\phi_{\tau}^{*}\right)^{\prime}(y)\right|}{\left|\left(\phi_{\tau}^{*}\right)^{\prime}(x)\right|} & =\frac{\left|\left(\phi_{\omega} \circ \phi_{i}\right)^{\prime}(y)\right|}{\left|\left(\phi_{\omega} \circ \phi_{i}\right)^{\prime}(x)\right|}=\frac{\left|\phi_{\omega}^{\prime}\left(\phi_{i}(y)\right)\right|}{\left|\phi_{\omega}^{\prime}\left(\phi_{i}(x)\right)\right|} \cdot \frac{\left|\phi_{i}^{\prime}(y)\right|}{\left|\phi_{i}^{\prime}(x)\right|} \\
& \leq L_{5}^{\left|\phi_{i}(y)-\phi_{i}(x)\right|^{\theta}} \exp \left(\frac{\left|\phi_{i}^{\prime}(y)\right|}{\left|\phi_{i}^{\prime}(x)\right|}-1\right) \\
& \leq L_{5}^{|y-x|^{\theta}} \exp \left(\frac{\left\|\Phi^{\prime} \mid\right\|_{\theta}}{u}|y-x|^{\theta}\right) \\
& \leq L_{5}^{|y-x|^{\theta}} \exp \left(\frac{M}{u}|y-x|^{\theta}\right)
\end{aligned}
$$

and the proof is finished.

The pressure function $P_{\Phi}(t)$ for the parabolic IFS $\Phi$ is defined by

$$
P_{\Phi}(t)=\lim _{n \rightarrow \infty} \frac{1}{n} \sum_{|\omega|=n}\left\|\phi_{\omega}^{\prime}\right\|^{t},
$$

in accordance with (2.3). Observe that $P_{\Phi}(0)=\log k$. In contrast with the hyperbolic case, $P_{\Phi}(t) \geq 0$ for all $t>0$ since $\left\|\phi_{k^{n}}^{\prime}\right\|=1$ for all $n$. It is easy to see that $P_{\Phi}(t)$ is non-increasing and continuous. Thus, $P_{\Phi}(s)=0$ implies $P_{\Phi}(t)=0$ for all $t>s$. Denote

$$
s(\Phi)=\min \left\{t>0: P_{\Phi}(t)=0\right\} .
$$


If the pressure function has no zeros, we let $s(\Phi)=\infty$, however, the next proposition implies that $s(\Phi)<\infty$ for any $\Phi \in \Gamma_{X}(\theta)$.

Proposition 5.10. Let $\Phi \in \Gamma_{X}(\theta)$ and let $\Phi_{*}$ be the associated infinite hyperbolic IFS. Then

(i) $\Theta\left(\Phi_{*}\right)=\frac{\beta}{\beta+1}$ and $\Phi_{*}$ is regular, i.e., there exists a unique $s\left(\Phi_{*}\right)>$ $\Theta\left(\Phi_{*}\right)$ such that $P_{\Phi_{*}}\left(s\left(\Phi_{*}\right)\right)=0$;

(ii) $s\left(\Phi_{*}\right)=s(\Phi)$.

Before the proof, we point out the following.

Corollary 5.11. If $\Phi \in \Gamma_{X}(\theta)$ then

(i) $\Phi_{*} \in \Xi_{X}\left(K_{1}, \gamma, \theta\right)$ where $K_{1}$ is from Corollary 5.9 ;

(ii) $\operatorname{dim}_{\mathrm{H}}\left(J_{\Phi}\right) \leq s(\Phi)$.

Proof. (i) follows from Proposition 5.10(i) and Definition 2.2.

(ii) follows from Proposition 5.10(ii), Lemma 2.3, and Corollary 5.7.

Proof of Proposition 5.10. (i) First we compute $\Theta\left(\Phi_{*}\right)$, see (2.6) for the definition. It follows from (5.1) and Lemma 5.3 that there exists a constant $K_{2}>1$ such that for all $x \in X$ and $b=(l, i) \in I$,

$$
\left|\left(\phi_{b}^{*}\right)^{\prime}(x)\right|=\left|\left(\phi_{k}^{l} \phi_{i}\right)^{\prime}(x)\right| \in\left(K_{2}^{-1}, K_{2}\right)(l+1)^{-\frac{\beta+1}{\beta}} .
$$

Therefore,

$$
\left|\left(\phi_{b}^{*}\right)^{\prime}(x)\right| \geq K_{2}^{-2}\left\|\left(\phi_{b}^{*}\right)^{\prime}\right\| \quad \text { for all } x \in X .
$$

Let $t>\frac{\beta}{\beta+1}$. We have by (5.14), writing $\phi_{\tau}^{*}=\phi_{\tau_{1}}^{*} \ldots \phi_{\tau_{n}}^{*}$ :

$$
\begin{aligned}
Z_{n}\left(\Phi_{*}, t\right)=\sum_{|\tau|=n}\left\|\left(\phi_{\tau}^{*}\right)^{\prime}\right\|^{t} & \in\left(K_{2}^{-2 t n}, 1\right) \sum_{|\tau|=n}\left\|\left(\phi_{\tau_{1}}^{*}\right)^{\prime}\right\|^{t} \ldots\left\|\left(\phi_{\tau_{n}}^{*}\right)^{\prime}\right\|^{t} \\
& =\left(K_{2}^{-2 t n}, 1\right)\left(\sum_{b \in I}\left\|\left(\phi_{b}^{*}\right)^{\prime}\right\|^{t}\right)^{n} \\
& =\left(K_{2}^{-2 t n}, 1\right)\left(\sum_{i \leq k-1} \sum_{l \geq 0}\left\|\left(\phi_{k}^{l} \phi_{i}\right)^{\prime}\right\|^{t}\right)^{n} .
\end{aligned}
$$

Next we use (5.13) to get

$$
\begin{aligned}
Z_{n}\left(\Phi_{*}, t\right) & \in\left(K_{2}^{-3 t n}, K_{2}^{t n}\right)\left(\sum_{i \leq k-1} \sum_{l \geq 0}(l+1)^{-\frac{\beta+1}{\beta} t}\right)^{n} \\
& =\left(K_{2}^{-3 t n}(k-1)^{n}, K_{2}^{t n}(k-1)^{n}\right)\left(\sum_{l \geq 0}(l+1)^{-\frac{\beta+1}{\beta} t}\right)^{n} .
\end{aligned}
$$


Since $P_{\Phi_{*}}(t)=\lim _{n \rightarrow \infty} \frac{1}{n} \log Z_{n}\left(\Phi_{*}, t\right)$ we obtain

$$
\log (k-1)-3 t \log K_{2} \leq P_{\Phi_{*}}(t)-\sum_{l \geq 0}(l+1)^{-\frac{\beta+1}{\beta} t} \leq \log (k-1)+t \log K_{2} .
$$

It follows that $P_{\Phi_{*}}(t)<\infty$ for $t>\frac{\beta}{\beta+1}$ and $\lim _{t \searrow \frac{\beta}{\beta+1}} P_{\Phi_{*}}(t)=+\infty$. Thus, $\Theta\left(\Phi_{*}\right)=\frac{\beta}{\beta+1}$. Since $P_{\Phi_{*}}(t)$ is positive and finite for some $t$, we conclude from Lemma 2.1 that there exists a unique solution $s\left(\Phi_{*}\right)$ for the Bowen's equation $P_{\Phi_{*}}(t)=0$. Part (i) is proved.

(ii) First we observe that

$$
s\left(\Phi_{*}\right)=s^{\prime}:=\inf \left\{t>0: \sum_{n \geq 1} Z_{n}\left(\Phi_{*}, t\right)<\infty\right\} .
$$

Indeed, since $P_{\Phi_{*}}(t)$ is strictly decreasing, $s\left(\Phi_{*}\right)=\inf \left\{t>0: P_{\Phi_{*}}(t)<0\right\}$. If $P_{\Phi_{*}}(t)<-\epsilon<0$ then $Z_{n}\left(\Phi_{*}, t\right) \leq C e^{-\epsilon n}$ as $n \rightarrow \infty$ and so $t \geq s^{\prime}$. Thus, $s\left(\Phi_{*}\right) \geq s^{\prime}$. On the other hand, if $\sum_{n \geq 1} Z_{n}\left(\Phi_{*}, t\right)<\infty$ then $Z_{n}\left(\Phi_{*}, t\right) \rightarrow 0$ hence $P_{\Phi_{*}}(t) \leq 0$. Therefore, $s\left(\Phi_{*}\right) \leq s^{\prime}$ and (5.15) is proved.

Next we demonstrate that for all $t>0$,

$$
Z_{n}(\Phi, t) \leq 1+\sum_{m \leq n} Z_{m}\left(\Phi_{*}, t\right) .
$$

Indeed, every $\omega \in \mathcal{A}^{n}$ can be written as $\omega=k^{n}$ or

$$
\omega=k^{r_{1}} i_{1} k^{r_{2}} i_{2} \ldots k^{r_{l}} i_{l} k^{r_{l+1}}
$$

where $l \geq 1$ and $i_{p} \leq k-1, r_{p} \geq 0$, for $p \leq l+1$. Thus, either $\phi_{\omega}=\phi_{k}^{n}$ or $\phi_{\omega}=\phi_{\tau}^{*} \phi_{k}^{r_{l+1}}$ for some $\tau \in I^{*}$. In the latter case, $\left\|\phi_{\omega}^{\prime}\right\| \leq\left\|\left(\phi_{\tau}^{*}\right)^{\prime}\right\|$ and $|\tau|=l \leq n$. Moreover, every map $\phi_{\tau}^{*}$ with $|\tau| \leq n$ occurs in this procedure at most once. The estimate (5.16) follows by noting that $\left\|\phi_{k^{n}}^{\prime}\right\|=1$.

Now we can show that $s\left(\Phi_{*}\right) \geq s(\Phi)=\min \left\{t>0: P_{\Phi}(t)=0\right\}$. In fact, if $\sum_{n \geq 1} Z_{n}\left(\Phi_{*}, r\right)<\infty$ then $Z_{n}(\Phi, r)$ is bounded by (5.16) and hence

$$
P_{\Phi}(r)=\lim _{n \rightarrow \infty} \frac{1}{n} \log Z_{n}(\Phi, r) \leq 0 .
$$

Therefore, $r \geq s(\Phi)$ and the desired inequality follows from (5.15).

It remains to verify that $s\left(\Phi_{*}\right) \leq s(\Phi)$. Recall Lemma 2.4(i) which says that if $\Psi \in \Xi_{X}(K, \gamma, \theta)$ then for every $t>0$ and every $n \geq 1$,

$$
P_{\Psi}(t) \leq \frac{1}{n} \log Z_{n}(\Psi, t) \leq P_{\Psi}(t)+\frac{t \log K}{n} .
$$

It is enough to prove that if $r>s(\Phi)$ then $r \geq s\left(\Phi_{*}\right)$. We have $P_{\Phi}(r)=0$. Fix an arbitrary $\delta>0$. It suffices to show that $P_{\Phi_{*}}(r) \leq \delta$. It is convenient to set $\Psi:=\Phi_{*}$. By the first part of this proposition, $\Phi_{*} \in \Xi_{X}\left(K_{1}, \gamma, \theta\right)$. 
Take $q \in \mathbb{N}$ so large that $\frac{r \log K_{1}}{q}<\frac{\delta}{8}$. Then there exists a finite subset $F \subset I$ such that

$$
0<\frac{1}{q} \log Z_{q}(\Psi, r)-\frac{1}{q} \log Z_{q}\left(\Psi_{F}, r\right)<\frac{\delta}{4} .
$$

Now applying (5.17) to $\Psi$ and $\Psi_{F}$ (which is obviously in $\Xi_{X}\left(K_{1}, \gamma, \theta\right.$ ) as well) we obtain for all $n \geq q$ :

$$
0<\frac{1}{n} \log Z_{n}(\Psi, r)-\frac{1}{n} \log Z_{n}\left(\Psi_{F}, r\right)<\frac{\delta}{4}+\frac{2 r \log K_{1}}{q}<\frac{\delta}{2} .
$$

Recall that $\Psi_{F}$ is a finite IFS so its elements (which are of the form $\phi_{k}^{l} \phi_{i}$ ) have some finite maximal length, say $L$, over the alphabet $\mathcal{A}$. Therefore, $Z_{n}\left(\Psi_{F}, r\right) \leq \sum_{p \leq n L} Z_{p}(\Phi, r)$. Since $P_{\Phi}(r)=0$, there exists $m \in \mathbb{N}$ such that $Z_{p}(\Phi, r) \leq e^{\frac{p}{2 L}}$ for $p \geq m$. Then

$$
Z_{n}\left(\Psi_{F}, r\right) \leq \sum_{p \leq m} Z_{p}(\Phi, r)+\sum_{p=m+1}^{n L} e^{\frac{p \delta}{2 L}} \leq C\left(e^{\delta n / 2}\right) .
$$

This implies that $\lim _{n \rightarrow \infty} \frac{1}{n} \log Z_{n}\left(\Psi_{F}, r\right) \leq \frac{\delta}{2}$, and together with (5.18) this yields

$$
P_{\Psi}(r)=\lim _{n \rightarrow \infty} \frac{1}{n} \log Z_{n}(\Psi, r) \leq \frac{\delta}{2}+\frac{\delta}{2}=\delta,
$$

as desired. The proof is complete.

\section{Families of parabolic IFS.}

Let $U \subset \mathbb{R}^{d}$ be an open set. Here we consider families of parabolic IFS

$$
\Phi^{\mathbf{t}}=\left\{\phi_{1}^{\mathbf{t}}, \ldots, \phi_{k-1}^{\mathbf{t}}, \phi_{k}\right\}
$$

depending on $\mathbf{t} \in \bar{U}$, the closure of $U$. We assume that $\Phi^{\mathbf{t}} \in \Gamma_{X}(\theta)$ for all $\mathbf{t} \in \bar{U}$ (see Definition 5.5.). Although the parabolic function does not depend on the parameter, it is sometimes convenient to write $\phi_{k}^{\mathbf{t}} \equiv \phi_{k}$ for $\mathbf{t} \in U$. Let $\pi_{\mathbf{t}}: \mathcal{A}^{\infty} \rightarrow \mathbb{R}$ be the natural projection associated with $\Phi^{\mathbf{t}}$ and denote $J_{\mathbf{t}}=\pi_{\mathbf{t}}\left(\mathcal{A}^{\infty}\right)$. Two conditions which control the dependence on $\mathbf{t}$ will be needed.

Continuity: The maps

$$
\mathbf{t} \mapsto \phi_{i}^{\mathbf{t}} \text { are continuous from } \bar{U} \text { to } \mathcal{C}^{1+\theta}(X) \text { for } i \leq k-1 .
$$

Transversality Condition: There exists a constant $C_{1}^{\prime}$ such that for all $\omega$ and $\tau$ in $\mathcal{A}^{\infty}$ with $\omega_{1} \neq \tau_{1}$,

$$
\mathcal{L}_{d}\left\{\mathbf{t} \in U:\left|\pi_{\mathbf{t}}(\omega)-\pi_{\mathbf{t}}(\tau)\right| \leq r\right\} \leq C_{1}^{\prime} r \text { for all } r>0 .
$$


This condition is almost identical to the transversality condition (3.2) except that here $\mathcal{A}$ is finite so $C_{1}^{\prime}$ is an absolute constant. Let

$$
s(\mathbf{t})=s\left(\Phi^{\mathbf{t}}\right)=\min \left\{u>0: P_{\Phi^{\mathbf{t}}}(u)=0\right\} .
$$

The following theorem is the main result of the paper.

Theorem 6.1. Suppose that $\left\{\Phi^{\mathbf{t}}\right\}_{\mathbf{t} \in \bar{U}}$ is a family of parabolic IFS in $\Gamma_{X}(\theta)$ satisfying (6.1) and (6.2). Then the function $\mathbf{t} \mapsto s(\mathbf{t})$ is continuous on $U$ and

(i) $\operatorname{dim}_{\mathrm{H}}\left(J_{\mathbf{t}}\right)=\min \{s(\mathbf{t}), 1\}$ for Lebesgue-a.e. $\mathbf{t} \in U$;

(ii) $\mathcal{L}_{1}\left(J_{\mathbf{t}}\right)>0$ for Lebesgue-a.e. $\mathbf{t} \in U$ such that $s(\mathbf{t})>1$.

Proof. The plan is to apply Theorem 3.1 to the family of associated infinite hyperbolic IFS

$$
\Phi_{*}^{\mathbf{t}}=\left\{\phi_{k}^{n} \phi_{i}^{\mathbf{t}}: n \geq 0, i \leq k-1\right\} .
$$

Since the family $\left\{\Phi^{\mathbf{t}}\right\}_{\mathbf{t} \in \bar{U}}$ satisfies the continuity condition (6.1), we can find $V, \gamma, u, M$, so that

$$
\Phi^{\mathbf{t}} \in \Gamma_{X}(\theta, V, \gamma, u, M) \quad \text { for all } \mathbf{t} \in U,
$$

see (5.4) for the meaning of this notation. These numbers and the neighborhood $V$ of $v$ will be fixed for the rest of the proof.

Observe that the constant $K_{1}$ in Corollary 5.9 is independent of $\mathbf{t} \in U$ and by Corollary 5.11(i) we have $\Phi_{*}^{\mathbf{t}} \in \Xi_{X}\left(K_{1}, \gamma, \theta\right)$ for $\mathbf{t} \in U$. Notice also that $s(\mathbf{t})=s\left(\Phi_{*}^{\mathbf{t}}\right)$ by Proposition 5.10(ii). Thus, to prove the theorem it remains to verify the distortion continuity property (3.1) and transversality condition (3.2) for $\Phi_{*}^{\mathbf{t}}$. We begin with the latter since it is easier.

As in Section 5, the alphabet for the IFS $\Phi_{*}^{\mathbf{t}}$ will be $I=\{(n, i): n \geq$ $0, i \leq k-1\}$. Let $\zeta$ and $\xi$ be elements of $I^{\infty}$ with $\zeta_{1} \neq \xi_{1}$. To distinguish between the IFS $\Phi^{\mathbf{t}}$ and $\Phi_{*}^{\mathbf{t}}$ we denote by $\pi_{\mathbf{t}}^{*}: I^{\infty} \rightarrow \mathbb{R}$ the natural projection corresponding to the IFS $\Phi_{*}^{\mathbf{t}}$. Let $\zeta_{1}=(n, i)$ and $\xi_{1}=(m, j)$. Clearly,

$$
\pi_{\mathbf{t}}^{*}(\zeta)=\pi_{\mathbf{t}}(\omega), \quad \pi_{\mathbf{t}}^{*}(\xi)=\pi_{\mathbf{t}}(\tau)
$$

where $\omega \in \mathcal{A}^{\infty}$ begins with $k^{n} i$ and $\tau \in \mathcal{A}^{\infty}$ begins with $k^{m} j$. Assume without loss of generality that $n \leq m$. Then

$$
\pi_{\mathbf{t}}^{*}(\zeta)-\pi_{\mathbf{t}}^{*}(\xi)=\pi_{\mathbf{t}}(\omega)-\pi_{\mathbf{t}}(\tau)=\phi_{k}^{n}\left(\pi_{\mathbf{t}}\left(\sigma^{n} \omega\right)\right)-\phi_{k}^{n}\left(\pi_{\mathbf{t}}\left(\sigma^{n} \tau\right)\right)
$$

and $\left(\sigma^{n} \omega\right)_{1} \neq\left(\sigma^{n} \tau\right)_{1}$. Thus, by the Mean Value Theorem and (5.7), for some $c \in X$,

$$
\left|\pi_{\mathbf{t}}^{*}(\zeta)-\pi_{\mathbf{t}}^{*}(\xi)\right|=\left|\left(\phi_{k}^{n}\right)^{\prime}(c)\right| \cdot\left|\pi_{\mathbf{t}}\left(\sigma^{n} \omega\right)-\pi_{\mathbf{t}}\left(\sigma^{n} \tau\right)\right| \geq u^{n}\left|\pi_{\mathbf{t}}\left(\sigma^{n} \omega\right)-\pi_{\mathbf{t}}\left(\sigma^{n} \tau\right)\right| .
$$

Therefore, by (6.2),

$$
\begin{aligned}
& \mathcal{L}_{d}\left\{\mathbf{t} \in U:\left|\pi_{\mathbf{t}}^{*}(\zeta)-\pi_{\mathbf{t}}^{*}(\xi)\right| \leq r\right\} \\
& \leq \mathcal{L}_{d}\left\{\mathbf{t} \in U:\left|\pi_{\mathbf{t}}\left(\sigma^{n} \omega\right)-\pi_{\mathbf{t}}\left(\sigma^{n} \tau\right)\right| \leq r u^{-n}\right\} \\
& \leq C_{1}^{\prime} r u^{-n}
\end{aligned}
$$


which implies (3.2) with $C\left(\xi_{1}, \eta_{1}\right):=C_{1}^{\prime} u^{-n}$.

The property (3.1) for $\Phi_{*}^{\mathbf{t}}$ is immediate from (6.1) and the following lemma where we let

$$
\|\Phi-\Psi\|=\max _{i \leq k-1}\left\|\phi_{i}-\psi_{i}\right\| \quad \text { and } \quad\left\|\Phi^{\prime}-\Psi^{\prime}\right\|=\max _{i \leq k-1}\left\|\phi_{i}^{\prime}-\psi_{i}^{\prime}\right\| .
$$

Lemma 6.2. There exists a positive constant $C_{2}^{\prime}=C_{2}^{\prime}(X, \theta, V, \gamma, u, M)$ such that for any $\Phi=\left\{\phi_{1}, \ldots, \phi_{k}\right\}$ and $\Psi=\left\{\psi_{1}, \ldots, \psi_{k}\right\}$, two parabolic IFS in $\Gamma_{X}(\theta, V, \gamma, u, M)$ with $\phi_{k}=\psi_{k}$, the associated infinite hyperbolic IFS $\Phi_{*}$ and $\Psi_{*}$ have the following property. For any $\tau \in I^{*}$ and any $x \in X$,

$$
\frac{\left|\left(\phi_{\tau}^{*}\right)^{\prime}(x)\right|}{\left|\left(\psi_{\tau}^{*}\right)^{\prime}(x)\right|} \leq \exp \left(C_{2}^{\prime}|\tau|\left(\|\Phi-\Psi\|^{\theta}+\left\|\Phi^{\prime}-\Psi^{\prime}\right\|\right)\right) .
$$

Proof. Let $n=|\tau|$ and observe that for all $x \in X$ and $0 \leq j<n$ :

$$
\begin{aligned}
& \left|\phi_{\sigma^{j} \tau}^{*}(x)-\psi_{\sigma^{j} \tau}^{*}(x)\right| \\
& \leq\left|\phi_{\sigma^{j}\left(\left.\tau\right|_{n-1}\right)}^{*}\left(\phi_{\tau_{n}}^{*}(x)\right)-\phi_{\sigma^{j}\left(\left.\tau\right|_{n-1}\right)}^{*}\left(\psi_{\tau_{n}}^{*}(x)\right)\right| \\
& \quad+\left|\phi_{\sigma^{j}\left(\left.\tau\right|_{n-1}\right)}^{*}\left(\psi_{\tau_{n}}^{*}(x)\right)-\psi_{\sigma^{j}\left(\left.\tau\right|_{n-1}\right)}^{*}\left(\psi_{\tau_{n}}^{*}(x)\right)\right| \\
& \leq \gamma^{n-j-1}\left\|\Phi_{*}-\Psi_{*}\right\|+\left|\phi_{\sigma^{j}\left(\left.\tau\right|_{n-1}\right)}^{*}\left(x^{\prime}\right)-\psi_{\sigma^{j}\left(\left.\tau\right|_{n-1}\right)}^{*}\left(x^{\prime}\right)\right|,
\end{aligned}
$$

for $x^{\prime}=\psi_{\tau_{n}}^{*}(x)$. Proceeding inductively we obtain

$$
\left|\phi_{\sigma^{j} \tau}^{*}(x)-\psi_{\sigma^{j} \tau}^{*}(x)\right| \leq \sum_{i=1}^{n-j} \gamma^{n-i-1}\left\|\Phi_{*}-\Psi_{*}\right\|<\frac{\left\|\Phi_{*}-\Psi_{*}\right\|}{1-\gamma} .
$$

Observe that

$$
\left\|\Phi_{*}-\Psi_{*}\right\|=\max _{i \leq k-1, m \geq 0}\left\|\phi_{k}^{m} \phi_{i}-\phi_{k}^{m} \psi_{i}\right\|=\max _{i \leq k-1}\left\|\phi_{i}-\psi_{i}\right\|=\|\Phi-\Psi\|
$$

since $\left\|\left(\phi_{k}^{m}\right)^{\prime}\right\|=1$. Now let $y=\phi_{\sigma^{j} \tau}^{*}(x)$ and $z=\psi_{\sigma^{j} \tau}^{*}(x)$, and suppose that $\tau_{j}=(l, i)$. Then $\phi_{\tau_{j}}^{*}=\phi_{k}^{l} \phi_{i}$ and $\psi_{\tau_{j}}^{*}=\phi_{k}^{l} \psi_{i}$, and we can estimate by (5.9):

$$
\begin{aligned}
\log \left|\frac{\left(\psi_{\tau_{j}}^{*}\right)^{\prime}\left(\psi_{\sigma^{j} \tau}^{*}(x)\right)}{\left(\phi_{\tau_{j}}^{*}\right)^{\prime}\left(\phi_{\sigma^{j} \tau}^{*}(x)\right)}\right| & =\log \left|\frac{\left(\phi_{k}^{l} \psi_{i}\right)^{\prime}(z)}{\left(\phi_{k}^{l} \phi_{i}\right)^{\prime}(y)}\right|=\log \left|\frac{\left(\phi_{k}^{l}\right)^{\prime}\left(\psi_{i}(z)\right)}{\left(\phi_{k}^{l}\right)^{\prime}\left(\phi_{i}(y)\right)}\right|+\log \left|\frac{\psi_{i}^{\prime}(z)}{\phi_{i}^{\prime}(y)}\right| \\
& \leq \log L_{5} \cdot\left|\psi_{i}(z)-\phi_{i}(y)\right|^{\theta}+u^{-1}\left|\psi_{i}^{\prime}(z)-\phi_{i}^{\prime}(y)\right| .
\end{aligned}
$$

Next,

$$
\begin{aligned}
\left|\psi_{i}(z)-\phi_{i}(y)\right| & \leq\left|\psi_{i}(z)-\psi_{i}(y)\right|+\left|\psi_{i}(y)-\phi_{i}(y)\right| \\
& \leq|z-y|+\|\Phi-\Psi\| \leq\|\Phi-\Psi\| \frac{2-\gamma}{1-\gamma}
\end{aligned}
$$


by (6.4) and (6.5). Furthermore,

$$
\begin{aligned}
\left|\psi_{i}^{\prime}(z)-\phi_{i}^{\prime}(y)\right| & \leq\left|\psi_{i}^{\prime}(z)-\psi_{i}^{\prime}(y)\right|+\left|\psi_{i}^{\prime}(y)-\phi_{i}^{\prime}(y)\right| \\
& \leq\left\|\Psi^{\prime}\right\|_{\theta}|z-y|^{\theta}+\left\|\Phi^{\prime}-\Psi^{\prime}\right\| \\
& \leq M\|\Phi-\Psi\|^{\theta}(1-\gamma)^{-\theta}+\left\|\Phi^{\prime}-\Psi^{\prime}\right\| .
\end{aligned}
$$

Combining the last two estimates with (6.6) yields

$$
\begin{aligned}
\log \left|\frac{\left(\psi_{\tau_{j}}^{*}\right)^{\prime}\left(\psi_{\sigma^{j} \tau}^{*}(x)\right)}{\left(\phi_{\tau_{j}}^{*}\right)^{\prime}\left(\phi_{\sigma^{j} \tau}^{*}(x)\right)}\right| \\
\leq\|\Phi-\Psi\|^{\theta}\left(\frac{2-\gamma}{1-\gamma}\right)^{\theta} \log L_{5} \\
\quad+u^{-1}\left(M\|\Phi-\Psi\|^{\theta}(1-\gamma)^{-\theta}+\left\|\Phi^{\prime}-\Psi^{\prime}\right\|\right) \\
\leq C_{2}^{\prime}\left(\|\Phi-\Psi\|^{\theta}+\left\|\Phi^{\prime}-\Psi^{\prime}\right\|\right)
\end{aligned}
$$

for some constant $C_{2}^{\prime}=C_{2}^{\prime}(X, \theta, V, \gamma, u, M)$. Exchanging the roles of $\Phi$ and $\Psi$ we can see that

$$
|\log | \frac{\left(\psi_{\tau_{j}}^{*}\right)^{\prime}\left(\psi_{\sigma^{j} \tau}^{*}(x)\right)}{\left(\phi_{\tau_{j}}^{*}\right)^{\prime}\left(\phi_{\sigma^{j} \tau}^{*}(x)\right)}|| \leq C_{2}^{\prime}\left(\|\Phi-\Psi\|^{\theta}+\left\|\Phi^{\prime}-\Psi^{\prime}\right\|\right) .
$$

Finally,

$$
\begin{aligned}
|\log | \frac{\left(\psi_{\tau}^{*}\right)^{\prime}(x)}{\left(\phi_{\tau}^{*}\right)^{\prime}(x)} \| & =\left|\sum_{j=1}^{n} \log \right| \frac{\left(\psi_{\tau_{j}}^{*}\right)^{\prime}\left(\psi_{\sigma^{j} \tau}^{*}(x)\right)}{\left(\phi_{\tau_{j}}^{*}\right)^{\prime}\left(\phi_{\sigma^{j} \tau}^{*}(x)\right)}|| \\
& \leq \sum_{j=1}^{n} C_{2}^{\prime}\left(\|\Phi-\Psi\|^{\theta}+\left\|\Phi^{\prime}-\Psi^{\prime}\right\|\right) \\
& =n C_{2}^{\prime}\left(\|\Phi-\Psi\|^{\theta}+\left\|\Phi^{\prime}-\Psi^{\prime}\right\|\right) .
\end{aligned}
$$

The lemma is proved, and this also concludes the proof of Theorem 6.1.

Given $\omega \in \mathcal{A}^{*}$ let $h(\omega)$ denote the number of hyperbolic letters (i.e., $\neq k$ ) appearing in $\omega$. We record the following useful corollary for future reference although it is not needed in this paper.

Corollary 6.3. There exists a positive constant $C_{2}^{\prime}=C_{2}^{\prime}(X, \theta, V, \gamma, u, M)$ such that for any $\Phi=\left\{\phi_{1}, \ldots, \phi_{k}\right\}$ and $\Psi=\left\{\psi_{1}, \ldots, \psi_{k}\right\}$, two parabolic IFS in $\Gamma_{X}(\theta, V, \gamma, u, M)$ with $\phi_{k}=\psi_{k}$, for all $\omega \in \mathcal{A}^{*}$ and all $x \in X$,

$$
\frac{\left|\phi_{\omega}^{\prime}(x)\right|}{\left|\psi_{\omega}^{\prime}(x)\right|} \leq \exp \left(C_{2}^{\prime} h(\omega)\left(\|\Phi-\Psi\|^{\theta}+\left\|\Phi^{\prime}-\Psi^{\prime}\right\|\right)\right) .
$$


Proof. For any $\omega \in \mathcal{A}^{*}$ we can find $\tau \in I^{*}$ and $l \geq 0$ such that

$$
\phi_{\omega}=\phi_{\tau}^{*} \phi_{k}^{l} \quad \text { and } \quad \psi_{\omega}=\psi_{\tau}^{*} \phi_{k}^{l} .
$$

By Lemma 6.2 we have

$$
\begin{aligned}
\frac{\left|\phi_{\omega}^{\prime}(x)\right|}{\left|\psi_{\omega}^{\prime}(x)\right|} & =\frac{\left|\left(\phi_{\tau}^{*}\right)^{\prime}\left(\phi_{k}^{l}(x)\right)\left(\phi_{k}^{l}\right)^{\prime}(x)\right|}{\left|\left(\psi_{\tau}^{*}\right)^{\prime}\left(\phi_{k}^{l}(x)\right)\left(\phi_{k}^{l}\right)^{\prime}(x)\right|}=\frac{\left|\left(\phi_{\tau}^{*}\right)^{\prime}\left(\phi_{k}^{l}(x)\right)\right|}{\left|\left(\psi_{\tau}^{*}\right)^{\prime}\left(\phi_{k}^{l}(x)\right)\right|} \\
& \leq \exp \left(C_{2}^{\prime}|\tau|\left(\|\Phi-\Psi\|^{\theta}+\left\|\Phi^{\prime}-\Psi^{\prime}\right\|\right)\right)
\end{aligned}
$$

which finishes the proof since $|\tau|=h(\omega)$.

We conclude this section with some finer results concerning exceptional parameters in Theorem 6.1(i). These will turn out to be almost immediate consequences of the results obtained in Section 4. The strong transversality condition is formulated in the context of parabolic systems in the same manner as the strong transversality condition in Section 4. Recall that $N_{r}(F)$ is the minimal number of balls of radius $r$ needed to cover the set $F \subset \mathbb{R}^{d}$.

Strong Transversality Condition: There exists a constant $C_{1}^{\prime}$ such that for all $\omega$ and $\tau$ in $\mathcal{A}^{\infty}$ with $\omega_{1} \neq \tau_{1}$,

$$
N_{r}\left(\left\{\mathbf{t} \in U:\left|\pi_{\mathbf{t}}(\omega)-\pi_{\mathbf{t}}(\tau)\right| \leq r\right\}\right) \leq C_{1}^{\prime} r \text { for all } r>0 .
$$

The same arguments as used in the proof of Theorem 6.1 demonstrate that the strong transversality condition (6.7) for a parabolic IFS implies the strong transversality condition (4.1) for the associated hyperbolic system. Consequently, Theorem 4.3 and Corollary 4.4 respectively imply the following.

Theorem 6.4. Suppose that $\left\{\Phi^{\mathbf{t}}\right\}_{\mathbf{t} \in \bar{U}}$ is a d-parameter family of parabolic IFS in $\Gamma_{X}(\theta)$ satisfying (6.1) and (6.7). If $G$ is an arbitrary subset of $U$, then for every $\xi>0$ we have

$$
\operatorname{dim}_{\mathrm{H}}\left(\left\{\mathbf{t} \in G: \operatorname{dim}_{\mathrm{H}}\left(J_{t}\right)<\min \{\xi, s(\mathbf{t})\}\right\}\right) \leq \min \left\{\xi, \sup _{G} s(\mathbf{t})\right\}+d-1 .
$$

Corollary 6.5. Suppose that $\left\{\Phi^{\mathbf{t}}\right\}_{\mathbf{t} \in \bar{U}}$ is a d-parameter family of parabolic IFS in $\Gamma_{X}(\theta)$ satisfying (6.1) and (6.7). Then for every $\mathbf{t}_{0} \in U$ we have $\lim _{r \rightarrow 0} \operatorname{dim}_{\mathrm{H}}\left(\left\{t \in B\left(\mathbf{t}_{0}, r\right): \operatorname{dim}_{\mathrm{H}}\left(J_{\mathbf{t}}\right)<\min \{\xi, s(\mathbf{t})\}\right\}\right) \leq \min \left\{\xi, s\left(\mathbf{t}_{0}\right)\right\}+d-1$. 


\section{Examples.}

7.1. General classes of examples. We are going to apply Theorem 6.1 to two more specific types of IFS families. In each case we need to impose some bounds on derivatives to guarantee transversality (even strong transversality), which is the most difficult condition to check. Recall that $\|\cdot\|_{Y}$ denotes the supremum norm on $Y$.

Proposition 7.1. Let $\Phi=\left\{\phi_{1}, \ldots, \phi_{k}\right\} \in \Gamma_{X}(\theta)$ and $1 \leq d \leq k-1$. Assume that

$\phi_{i}$ are increasing for all $i \leq k$;

and

$$
\left\|\phi_{i}^{\prime}\right\|+\left\|\phi_{j}^{\prime}\right\|_{\phi_{j}^{-1} \phi_{i}(X)}<1 \text { for all } i<j \text { such that } \phi_{i}(X) \cap \phi_{j}(X) \neq \emptyset \text {. }
$$

Consider the family

$$
\begin{aligned}
\Phi^{\mathbf{t}}=\left\{\phi_{1}(x)+t_{1}, \ldots, \phi_{d}(x)+t_{d}, \phi_{d+1}(x)\right. & \left.\ldots, \phi_{k}(x)\right\} \\
& \text { where } \mathbf{t}=\left(t_{1}, \ldots, t_{d}\right) \in \mathbb{R}^{d} .
\end{aligned}
$$

Then there exists $\eta>0$ such that $\left\{\Phi^{\mathbf{t}}: \mathbf{t} \in B_{\eta}(\mathbf{0})\right\}$ satisfies all the hypotheses of Theorem 6.1. Moreover, the strong transversality condition (6.7) is satisfied.

Remark. It is not hard to find specific IFS satisfying the above proposition. Notice that non-trivial examples start with $k=3$. Indeed, if $k=2$ there is a dichotomy: the limit set is either connected or the projection map $\pi_{\Phi}: \mathcal{A}^{\infty} \rightarrow J_{\Phi}$ is one-to-one. In the latter case there are no "overlaps", so the dimension formula holds by [U]. In the former case $J_{\Phi}$ is an interval, so there is no question about its dimension or measure. To get a non-trivial example with $k=3$, one can take a parabolic function, say, $\phi_{3}(x)=\sin x$ on $[0, b]$, with $b<\pi / 2$, and add two more functions so that there is a "gap" and an "overlap". For instance, an increasing function $\phi_{2}$ may be chosen to satisfy $\phi_{2}(0)>\sin b$ and $\phi_{2}(b)=b$, and the increasing function $\phi_{1}$ to satisfy $\phi_{1}(0) \in(0, \sin b), \phi_{1}(b)<\phi_{2}(0)$. Consider a one-parameter family $\Phi^{t}=\left\{\phi_{1}(x)+t, \phi_{2}(x), \phi_{3}(x)\right\}$ and call $t$ admissible if these properties persist for $\Phi^{t}$. If $t$ is admissible, then the limit set has the convex hull equal to $[0, b]$. We can define $X=[0, b+\epsilon]$ for a small $\epsilon>0$, so that $\phi_{2}(X) \subset \operatorname{Int}(X)$. All the assumptions of Proposition 7.1 are satisfied, provided that the derivative of $\phi_{1}$ is sufficiently small, so the results of Section 6 apply.

Example 1. The family of IFS

$$
\Phi^{t}=\left\{0.01(x+1)^{2}+t, 0.1 x+(4.5 / 11) \pi, \sin x\right\} \quad \text { on }\left[0, \frac{5 \pi}{11}+\epsilon\right]
$$


satisfies all the conditions above for $t \in[0.3,0.97]$ if $\epsilon>$ is sufficiently small. (We are grateful to P. Hanus for correcting our original faulty example.)

In the next proposition all elements of the IFS are assumed to be of the form $\phi\left(x+a_{j}\right)$ for a single function $\phi$. Although this seems much more special than Proposition 7.1, it covers some interesting families, in particular, those which arise from a class of continued fractions.

Proposition 7.2. (i) Let $\Phi=\left\{\phi_{1}, \ldots, \phi_{k}\right\} \in \Gamma_{X}(\theta)$ and $1 \leq d \leq k-1$ be such that (7.2) is satisfied. We further assume that there exists a single increasing function $\phi \in \mathcal{C}^{1+\theta}$ on some interval $Y$ and $a_{i} \in \mathbb{R}, u>0$ such that

$$
\phi_{i}(x)=\phi\left(x+a_{i}\right), i \leq k,
$$

and

$$
\inf _{x \in Y}\left|\phi^{\prime}(x)\right| \geq u>0 .
$$

Let

$\Phi^{\mathbf{t}}=\left\{\phi_{1}\left(x+t_{1}\right), \ldots, \phi_{d}\left(x+t_{d}\right), \phi_{d+1}(x), \ldots, \phi_{k}(x)\right\}$

$$
\text { where } \mathbf{t}=\left(t_{1}, \ldots, t_{d}\right) \in \mathbb{R}^{d} \text {. }
$$

Denote by $\pi_{\mathbf{t}}$ the projection map corresponding to $\Phi^{\mathbf{t}}$. If there exists $\delta>0$ such that

$$
\left.\frac{\partial}{\partial t_{i}} \pi_{\mathbf{t}}(\xi)\right|_{\mathbf{t}=0}<1-\delta \quad \text { for all } \xi \in \mathcal{A}^{\infty} \text { and all } 1 \leq i \leq d,
$$

then there exists $\eta>0$ such that $\left\{\Phi^{\mathbf{t}}: \mathbf{t} \in B_{\eta}(\mathbf{0})\right\}$ satisfies all the hypotheses of Theorem 6.1. Moreover, the strong transversality condition (6.7) is satisfied.

(ii) Condition (7.6) holds if

$$
\left\|\phi_{i}^{\prime}\right\|<1 / 2 \text { for all } 1 \leq i \leq d .
$$

Example 2. Let $\Phi^{a}=\left\{\ln (x+a), \ln \left(x+e^{2}-2\right), \ln (x+1)\right\}$. This is a parabolic IFS on $[0,2]$. We have $\left\|\phi_{1}^{\prime}\right\|=1 / a$, and so Proposition 7.2 and Theorem 6.1 imply that the dimension formula holds for a.e. $a \in\left(2, e^{2}-2\right)$. (We need to enlarge the interval slightly and let $X=[0,2+\epsilon]$ so that $\phi_{2}(X) \subset \operatorname{Int}(X)$.)

Turning to the proofs of Propositions 7.1 and 7.2, note that checking strong transversality (6.7) is the only issue since all other properties obviously hold for sufficiently small perturbations of $\Phi$. This will be done with the help of the following elementary lemma. 
Lemma 7.3. Let $U \subset \mathbb{R}^{d}$ be an open, bounded set with smooth boundary. Suppose that $f$ is a $\mathcal{C}^{1}$ real-valued function in a neighborhood of $\bar{U}$ such that for some $i \in\{1, \ldots, d\}$ there exists $\eta>0$ satisfying

$$
\mathbf{t} \in U, \quad|f(\mathbf{t})| \leq \eta \Longrightarrow \frac{\partial f(\mathbf{t})}{\partial t_{i}} \geq \eta
$$

Then there exists $C=C(\eta)$ such that

$$
N_{r}(\{\mathbf{t} \in U:|f(\mathbf{t})| \leq r\}) \leq C r, \quad \forall r>0 .
$$

Proof. Recall that $N_{r}(F)$ denotes the minimal number of balls of radius $r$ needed to cover $F$. Since $U$ is bounded it suffices to prove our lemma for every $r<\eta$. Let $M=f^{-1}(0)$. By $(7.8), \operatorname{grad}(f)(\mathbf{t}) \neq 0$ for every $\mathbf{t} \in M$. Hence $M$ is a $(d-1)$-dimensional $\mathcal{C}^{1}$-manifold and $(M \cap \bar{U}) \cup \partial U$ is contained in a union of finitely many compact connected $\mathcal{C}^{1}$ manifolds with smooth boundaries. Thus there exists a constant $C_{1}$ such that for every $r<\eta$ the set $(M \cap \bar{U}) \cup \partial U$ can be covered by $C_{1} r^{1-d}$ balls with radii $r$. In order to complete the proof it is sufficient to show that for each point $\mathbf{t}=\left(t_{1}, t_{2}, \ldots, t_{d}\right) \in U$ with $|f(\mathbf{t})| \leq r$, the distance between $\mathbf{t}$ and $(M \cap \bar{U}) \cup \partial U$ does not exceed $r / \eta$. Indeed, we will then cover the set $f^{-1}([-r, r])$ by at most $C_{1} r^{1-d}$ balls with radii $(1+1 / \eta) r$. But each ball of radius $(1+1 / \eta) r$ can be covered by $C_{2}(\eta)$ balls of radii $r$, where $C_{2}(\eta)$ depends only on $\eta$ and the dimension $d$ and we would therefore be done.

Without loss of generality let $i=1$. Consider the function $g(s)=$ $f\left(s, t_{2}, \ldots, t_{d}\right)$ defined in a neighborhood of $t_{1}$. Suppose first that $g\left(t_{1}\right) \in$ $[-r, 0]$. By (7.8), the function $g(s)$ increases for $s>t_{1}$ until either the point $\left(s, t_{2}, \ldots, t_{d}\right)$ reaches the boundary of $U$, say at a point $\left(u, t_{2}, \ldots, t_{d}\right) \in \partial U$ and $g(s) \in[-r, 0]$ for all $s \in\left[t_{1}, u\right]$, or $g(s)$ will take on the value zero earlier. Suppose that the first case materializes. Then $g^{\prime}(s) \geq \eta$ for every $s \in\left[t_{1}, u\right]$ by (7.8). By the Mean Value Theorem, $\left|u-t_{1}\right| \leq r / \eta$, and therefore, $\operatorname{dist}(\mathbf{t}, \partial U) \leq \operatorname{dist}\left(\mathbf{t},\left(u, t_{2}, \ldots, t_{d}\right)\right)=\left|u-t_{1}\right| \leq r / \eta$. So, we are done in this case. If the second case holds, let $w$ be that point for which $g(w)=0$ and $g(s) \in[-r, 0]$ for every $s \in\left[t_{1}, w\right]$. Then $\left(w, t_{2}, \ldots, t_{d}\right) \in M$ and $g^{\prime}(s) \geq \eta$ for every $s \in\left[t_{1}, w\right]$. Again by the Mean Value Theorem, $\left|w-t_{1}\right| \leq r / \eta$. Then $\operatorname{dist}(\mathbf{t}, M \cap \bar{U}) \leq \operatorname{dist}\left(\mathbf{t},\left(w, t_{2}, \ldots, t_{d}\right)\right)=\left|w-t_{1}\right| \leq r / \eta$ and we are done in this case as well. If $g\left(t_{1}\right) \in[0, r]$, we proceed similarly letting $s$ go left from $t_{1}$. The proof is complete.

Proof of Proposition 7.1. It is convenient to write $\Phi^{\mathbf{t}}=\left\{\phi_{i}^{\mathbf{t}}\right\}_{i \leq k}$. The properties (7.3) and (5.1) persist under a small perturbation, so we can find $\eta \in(0,1)$ such that

$$
\inf \left\{\operatorname{dist}\left(\phi_{i}^{\mathbf{t}}(X), v\right): i \leq k-1,\|\mathbf{t}\| \leq \eta\right\} \geq \eta>0 ;
$$


(7.11) $\inf \left\{\operatorname{dist}\left(\phi_{i}^{\mathbf{t}}(X), \phi_{j}^{\mathbf{t}}(X)\right): \phi_{i}(X) \cap \phi_{j}(X)=\emptyset,\|\mathbf{t}\| \leq \eta\right\} \geq \eta>0$

$\operatorname{dist}\left(\phi_{i}(X), \phi_{j}(x)\right) \leq 3 \eta \Longrightarrow\left\|\phi_{i}^{\prime}\right\|+\left|\phi_{j}^{\prime}(x)\right|<1-\eta \quad$ for $i \leq d, i<j$.

The continuity property (6.1) is obvious so we only need to verify the strong transversality condition (6.7).

Consider $\omega, \tau \in \mathcal{A}^{\infty}$, with $\omega_{1} \neq \tau_{1}$. Let $i=\omega_{1}$ and $j=\tau_{1}$. Without loss of generality assume that $i<j$. If $\phi_{i}(X) \cap \phi_{j}(X)=\emptyset$ then

$$
\left|\pi_{\mathbf{t}}(\omega)-\pi_{\mathbf{t}}(\tau)\right|=\left|\phi_{i}^{\mathbf{t}}\left(\pi_{\mathbf{t}}(\sigma \omega)\right)-\phi_{j}^{\mathbf{t}}\left(\pi_{\mathbf{t}}(\sigma \tau)\right)\right| \geq \eta>0
$$

for $\mathbf{t} \in B_{\eta}(\mathbf{0})$ by $(7.11)$ and

$$
\left\{\mathbf{t} \in B_{\eta}(\mathbf{0}):\left|\pi_{\mathbf{t}}(\omega)-\pi_{\mathbf{t}}(\tau)\right| \leq r\right\} \subset B_{r}(\mathbf{0})
$$

(note that the left-hand side is empty for $r<\eta$ ). Thus (6.7) certainly holds in this case. If $\phi_{i}(X) \cap \phi_{j}(X) \neq \emptyset$ then $i \leq d$ and

$$
\pi_{\mathbf{t}}(\omega)-\pi_{\mathbf{t}}(\tau)=t_{i}+\phi_{i}\left(\pi_{\mathbf{t}}(\sigma \omega)\right)-\phi_{j}^{\mathbf{t}}\left(\pi_{\mathbf{t}}(\sigma \tau)\right) .
$$

We are going to use Lemma 7.3 with $f(\mathbf{t})=\pi_{\mathbf{t}}(\omega)-\pi_{\mathbf{t}}(\tau)$ and $U=B_{\eta}(\mathbf{0})$, so we need to check (7.8). Since $\left|\phi_{j}^{\mathbf{t}}\left(\pi_{\mathbf{t}}(\sigma \tau)\right)-\phi_{j}\left(\pi_{\mathbf{t}}(\sigma \tau)\right)\right|=\left|t_{j}\right| \leq \eta$ and $\left|t_{i}\right| \leq \eta$, we have the implication

$$
\begin{aligned}
|f(\mathbf{t})|=\left|\pi_{\mathbf{t}}(\omega)-\pi_{\mathbf{t}}(\tau)\right| \leq \eta & \Longrightarrow\left|\phi_{i}\left(\pi_{\mathbf{t}}(\sigma \omega)\right)-\phi_{j}\left(\pi_{\mathbf{t}}(\sigma \tau)\right)\right| \leq 3 \eta \\
& \Longrightarrow\left\|\phi_{i}^{\prime}\right\|+\left|\phi_{j}^{\prime}\left(\pi_{\mathbf{t}}(\sigma \tau)\right)\right|<1-\eta
\end{aligned}
$$

by (7.12). Since $j \neq i$ we have

$$
\begin{aligned}
\frac{\partial}{\partial t_{i}}\left(\pi_{\mathbf{t}}(\omega)-\pi_{\mathbf{t}}(\tau)\right) & =1+\frac{\partial}{\partial t_{i}} \phi_{i}\left(\pi_{\mathbf{t}}(\sigma \omega)\right)-\frac{\partial}{\partial t_{i}} \phi_{j}\left(\pi_{\mathbf{t}}(\sigma \tau)\right) \\
& =1+\phi_{i}^{\prime}\left(\pi_{\mathbf{t}}(\sigma \omega)\right) \cdot \frac{\partial}{\partial t_{i}} \pi_{\mathbf{t}}(\sigma \omega)-\phi_{j}^{\prime}\left(\pi_{\mathbf{t}}(\sigma \tau)\right) \cdot \frac{\partial}{\partial t_{i}} \pi_{\mathbf{t}}(\sigma \tau) .
\end{aligned}
$$

Observe that $\phi_{i}^{\prime}>0$ and $\frac{\partial}{\partial t_{i}} \pi_{\mathbf{t}}(\sigma \omega) \geq 0$ by the assumption (7.1). Suppose that $(\sigma \tau)_{n}$ is the first symbol $i$ in $\sigma \tau$ (if $\sigma \tau$ contains no $i$ we have $\frac{\partial}{\partial t_{i}} \pi_{\mathbf{t}}(\sigma \tau)=$ 0 and the claim is obvious). Then

$$
\pi_{\mathbf{t}}(\sigma \tau)=\phi_{\left.\sigma \tau\right|_{n-1}}^{\mathbf{t}}\left(t_{i}+\phi_{i}\left(\pi_{\mathbf{t}}\left(\sigma^{n+1} \tau\right)\right)\right)
$$

and since $\left\|\left(\phi_{\left.\sigma \tau\right|_{n-1}}^{\mathbf{t}}\right)^{\prime}\right\| \leq 1$ we obtain

$$
\frac{\partial}{\partial t_{i}} \pi_{\mathbf{t}}(\sigma \tau) \leq 1+\left\|\phi_{i}^{\prime}\right\| \cdot \frac{\partial}{\partial t_{i}} \pi_{\mathbf{t}}\left(\sigma^{n+1} \tau\right)
$$

Proceeding inductively we see that

$$
\frac{\partial}{\partial t_{i}} \pi_{\mathbf{t}}(\sigma \tau) \leq 1+\left\|\phi_{i}^{\prime}\right\|+\left\|\phi_{i}^{\prime}\right\|^{2}+\ldots=\left(1-\left\|\phi_{i}^{\prime}\right\|\right)^{-1}
$$


(recall that $\phi_{i}$ is hyperbolic since $i \leq d<k$ ). Together with (7.15) this implies

$$
\begin{aligned}
\frac{\partial}{\partial t_{i}}\left(\pi_{\mathbf{t}}(\omega)-\pi_{\mathbf{t}}(\tau)\right) & \geq 1-\left|\phi_{j}^{\prime}\left(\pi_{\mathbf{t}}(\sigma \tau)\right)\right|\left(1-\left\|\phi_{i}^{\prime}\right\|\right)^{-1} \\
& =\frac{1-\left\|\phi_{i}^{\prime}\right\|-\left|\phi_{j}^{\prime}\left(\pi_{\mathbf{t}}(\sigma \tau)\right)\right|}{1-\left\|\phi_{i}^{\prime}\right\|}>\eta
\end{aligned}
$$

by (7.14). We have verified (7.8), so the strong transversality condition (6.7) holds by Lemma 7.3 and the proof is complete.

Proof of Proposition 7.2. is similar to that of Proposition 7.1. We can choose $\eta>0$ so that (7.10) and (7.11) hold, and moreover,

$$
\frac{\partial}{\partial t_{i}} \pi_{\mathbf{t}}(\xi)<1-\eta \quad \text { for all } \xi \in \mathcal{A}^{\infty}, i \leq d, \mathbf{t} \in B_{\eta}(\mathbf{0})
$$

Again we only need to check strong transversality. Let $\omega, \tau \in \mathcal{A}^{\infty}$ with $i=\omega_{1}, j=\tau_{1}$, and $i<j$. If $\phi_{i}(X) \cap \phi_{j}(X)=\emptyset$ we immediately obtain (7.13) by (7.11). If $\phi_{i}(X) \cap \phi_{j}(X) \neq \emptyset$ then $i \leq d$ and

$$
\begin{aligned}
\pi_{\mathbf{t}}(\omega)-\pi_{\mathbf{t}}(\tau) & =\phi_{i}\left(t_{i}+\pi_{\mathbf{t}}(\sigma \omega)\right)-\phi_{j}^{\mathbf{t}}\left(\pi_{\mathbf{t}}(\sigma \tau)\right) \\
& =\phi\left(a_{i}+t_{i}+\pi_{\mathbf{t}}(\sigma \omega)\right)-\phi\left(a_{j}+\kappa_{j} t_{j}+\pi_{\mathbf{t}}(\sigma \tau)\right) \\
& =\phi^{\prime}(c)\left(A\left(t_{j}\right)+t_{i}+\pi_{\mathbf{t}}(\sigma \omega)-\pi_{\mathbf{t}}(\sigma \tau)\right)
\end{aligned}
$$

where $\kappa_{j}=1$ if $j \leq d$ and 0 otherwise, and $A\left(t_{j}\right)=a_{i}-a_{j}-\kappa_{j} t_{j}$ does not depend on $t_{i}$. Denoting $f(\mathbf{t}):=A\left(t_{j}\right)+t_{i}+\pi_{\mathbf{t}}(\sigma \omega)-\pi_{\mathbf{t}}(\sigma \tau)$ we have by (7.5):

$$
\left\{\mathbf{t} \in B_{\eta}(\mathbf{0}):\left|\pi_{\mathbf{t}}(\omega)-\pi_{\mathbf{t}}(\tau)\right| \leq r\right\} \subset\left\{\mathbf{t} \in B_{\eta}(\mathbf{0}):|f(\mathbf{t})| \leq u^{-1} r\right\} .
$$

If we show that $\frac{\partial f}{\partial t_{i}} \geq \eta$ then strong transversality will follow from Lemma 7.3. Since all $\phi_{j}$ are increasing it is easy to see that $\frac{\partial}{\partial t_{i}} \pi_{\mathbf{t}}(\sigma \omega) \geq 0$, hence

$$
\frac{\partial f}{\partial t_{i}} \geq 1-\frac{\partial}{\partial t_{i}} \pi_{\mathbf{t}}(\sigma \tau)
$$

and the desired statement follows from (7.17).

(ii) It remains to derive (7.6) from (7.7). Let $1 \leq i \leq d$. If $\xi \in \mathcal{A}^{\infty}$ contains no $i$, then $\frac{\partial}{\partial t_{i}} \pi_{\mathbf{t}}(\xi)=0$. Otherwise we write $\xi=w i \widetilde{\xi}$ where $w \in \mathcal{A}^{*}$ contains no $i$ (the word $w$ may be empty). Then

$$
\pi_{\mathbf{t}}(\xi)=\phi_{w}^{\mathbf{t}} \phi_{i}\left(t_{i}+\pi_{\mathbf{t}}(\widetilde{\xi})\right)
$$

hence

$$
\frac{\partial}{\partial t_{i}} \pi_{\mathbf{t}}(\xi) \leq\left\|\left(\phi_{w}^{\mathbf{t}}\right)^{\prime}\right\| \cdot\left\|\phi_{i}^{\prime}\right\|\left(1+\frac{\partial}{\partial t_{i}} \pi_{\mathbf{t}}(\widetilde{\xi})\right) \leq\left\|\phi_{i}^{\prime}\right\|\left(1+\frac{\partial}{\partial t_{i}} \pi_{\mathbf{t}}(\widetilde{\xi})\right) .
$$


Proceeding inductively we obtain that

$$
\frac{\partial}{\partial t_{i}} \pi_{\mathbf{t}}(\widetilde{\xi}) \leq \sum_{n=1}^{\infty}\left\|\phi_{i}^{\prime}\right\|^{n}=1-\frac{1-2\left\|\phi_{i}^{\prime}\right\|}{1-\left\|\phi_{i}^{\prime}\right\|}<1-\delta
$$

for some $\delta>0$ by (7.7), and the proof is complete.

7.2. A class of continued fractions. Here we study in detail IFS arising from the function $\phi(x)=\frac{x}{x+1}$. Let $A=\left\{a_{1}, \ldots, a_{k}\right\}$ where $a_{i}>0$ for $i \leq k-1$ and $a_{k}=0$. Let $\Phi_{A}=\left\{\phi\left(x+a_{i}\right)\right\}_{i \leq k}$. Then $\phi_{k}=\phi$ is parabolic on $[0,1]$, with the neutral fixed point $v=0$, for all $A$. The functions $\phi\left(x+a_{i}\right)$, for $i \leq k-1$, are hyperbolic on $[0,1]$. Clearly, the IFS $\Phi_{A}$ belongs to $\Gamma_{[0,1]}(1)$ (see Definition 5.5). The IFS $\Phi_{A}$ with $A=\{\alpha, 0\}$ was recently considered by Lyons $[\mathbf{L y}]$ who studied the properties of the measure which arises from applying the maps randomly and independently with equal probabilities. However, as far as the limit set is concerned, the case $k=2$ is trivial, since then either the limit set is an interval, or the IFS has no overlaps, so the dimension formula holds for all parameters by [U].

The connection with continued fractions is as follows: The limit set $J_{A}$ of $\Phi_{A}$ on $[0,1]$ coincides with the set of continued fractions of the form

$$
y=\left[1, Y_{1}, 1, Y_{2}, 1, Y_{3}, 1, \ldots\right]:=\frac{1}{1+\frac{1}{Y_{1}+\frac{1}{1+\ldots}}}
$$

where $Y_{i} \in A$, and also with the set of continued fractions of the form

$$
y=1-\frac{1}{\left(2+Y_{1}\right)-\frac{1}{\left(2+Y_{2}\right)-\frac{1}{\left(2+Y_{3}\right)-\ldots}}}
$$

where $Y_{i} \in A$. Indeed, these representations are immediate by writing

$$
\frac{x+\alpha}{x+\alpha+1}=\frac{1}{1+\frac{1}{\alpha+x}} \quad \text { and } \quad \frac{x+\alpha}{x+\alpha+1}=1-\frac{1}{(1+\alpha)+x} \text {. }
$$

Example 3. Let $A=\{a, 2,0\}$. We start with the one-parameter family of IFS $\Phi_{A}$ corresponding to $a \in(0,2)$, however, we will see that the non-trivial interval of parameters is smaller.

First observe that the limit set of the IFS $\{\phi(x+a), \phi(x)\}$ is an interval if $a \in(0,1 / 2]$. Let $\phi_{1}(x)=\phi(x+a)$. Then $\phi_{1}(b)=b$ where $b=\frac{1}{2}(-a+$ $\left.\sqrt{a^{2}+4 a}\right)$. This implies that $[0, b] \supset \phi_{1}([0, b]) \cup \phi([0, b])$, and this becomes equality if and only if $\phi(b) \geq \phi_{1}(0)$ which is equivalent to $a \leq 1 / 2$. Since the limit set can only increase when more functions are added, $J_{A}$ contains an interval if $a \leq 1 / 2$. 
Since $\phi_{2}(x)=\phi(x+2)$ has the fixed point at $\sqrt{3}-1$, we see that $Y:=$ $[0, \sqrt{3}-1]$ is the convex hull of $J_{A}$. We have, denoting $\phi_{3}=\phi$,

$$
\begin{aligned}
& \phi_{1}(Y)=\left[\frac{a}{a+1}, \frac{\sqrt{3}-1+a}{\sqrt{3}+a}\right], \\
& \phi_{2}(Y)=[2 / 3, \sqrt{3}-1], \\
& \phi_{3}(Y)=[0,1-1 / \sqrt{3}] .
\end{aligned}
$$

Now, $\phi_{2}(Y) \cap \phi_{3}(Y)=\emptyset$. We want to make sure that the IFS has both a "gap" and an "overlap". Note that $\phi_{1}(Y) \cap \phi_{2}(Y) \neq \emptyset$ if and only if $a \in[3-\sqrt{3}, 2)$ and $\phi_{1}(Y) \cap \phi_{3}(Y) \neq \emptyset$ if and only if $a \in(0, \sqrt{3}-1]$ (recall that we only consider $0<a<2)$. Thus the "interesting" set of parameters is $U:=\left(\frac{1}{2}, \sqrt{3}-1\right) \cap(3-\sqrt{3}, 2)$.

We want to apply Proposition 7.2 with $k=3$ and $d=1$. Let $X=$ $[0, \sqrt{3}-1+\epsilon]$ where $\epsilon$ is so small that $\phi_{2}(X) \cap \phi_{3}(X)=\emptyset$. We have

$$
\left\|\phi_{1}^{\prime}\right\|=\phi_{1}^{\prime}(0)=(1+a)^{-2}<4 / 9<1 / 2,
$$

since $a>\frac{1}{2}$. Thus (7.7) holds and all the assumptions of Proposition 7.2 are satisfied. Let $s(a)=s\left(\Phi_{A}\right)$ where $A=\{a, 2,0\}$; then $s: U \rightarrow \mathbb{R}$ is continuous. Let $U_{<1}=\{A \in U: s(A)<1\}$ and $U_{>1}=\{A \in U: s(A)>1\}$. We obtain the following:

Corollary 7.4. (i) $\operatorname{dim}_{\mathrm{H}}\left(J_{A}\right)=\min \{s(a), 1\}$ for Lebesgue-a.e. $a \in U$.

(ii) For any set $G \subset U_{<1}$ we have

$$
\operatorname{dim}_{\mathrm{H}}\left\{a \in G: \operatorname{dim}_{\mathrm{H}}\left(J_{A}\right)<s(a)\right\} \leq \sup _{G} s(a) .
$$

(iii) $J_{A}$ has positive Lebesgue measure for Lebesgue-a.e. $a \in U_{>1}$.

Note that both $U_{<1}$ and $U_{>1}$ are non-empty. Indeed, continuity of $s(a)$ was established independent of transversality (see Lemma 3.4). Thus $s(a)$ is continuous on $(0,2)$. For $a=\frac{1}{2}$ we know that the limit set of the IFS $\left\{\phi_{1}, \phi_{3}\right\}$ is an interval. Since adding an extra function to the IFS makes $s(a)$ strictly larger, we see that $s\left(\frac{1}{2}\right)>1$. By continuity, this implies that $U_{>1} \neq \emptyset$. On the other hand, for $a=\sqrt{3}-1$ and for $a=3-\sqrt{3}$ the IFS satisfies the open set condition, as the interiors of $\phi_{i}(Y), i=1,2,3$, are disjoint. Since the limit set is disconnected at these parameter values, it follows, as in [U, Th.6.5], that $s(\sqrt{3}-1)<1$ and $s(3-\sqrt{3})<1$. By continuity, this implies that $U_{<1} \neq \emptyset$.

Example 4. Consider $\Phi_{A}=\left\{\phi\left(x+a_{i}\right)\right\}_{i \leq k}$ where

$$
A \in U:=\left\{A \in \mathbb{R}^{k}: a_{k}=0, a_{j}>0, j=1, \ldots, k-1\right\}, \quad k \geq 3 .
$$

This is a $(k-1)$-parameter family of parabolic IFS. 
Corollary 7.5. (i) For Lebesgue-a.e. $A \in U$,

$$
\operatorname{dim}_{\mathrm{H}}\left(J_{A}\right)=\min \{s(A), 1\} .
$$

(ii) For any set $G \subset U_{<1}$ we have

$$
\operatorname{dim}_{\mathrm{H}}\left\{A \in G: \operatorname{dim}_{\mathrm{H}}\left(J_{A}\right)<s(A)\right\} \leq \sup _{G} s(A)+(k-2) .
$$

(iii) For Lebesgue-a.e. $A \in U_{>1}$ the set $J_{A}$ has positive Lebesgue measure.

Proof. As in Example 3, we have that if $a_{i} \leq \frac{1}{2}$ for some $i \leq k-1$, then $J_{A}$ contains an interval an and there is nothing to prove. If $a_{i}>\frac{1}{2}$ for all $i$, then (7.7) holds. Then we can apply Proposition 7.2 (with $d=k-1$, when (7.2) is vacuous), and the statements follow from our theorems.

\section{References}

[B1] T. Bedford, Applications of dynamical systems theory to fractals - a study of cookie-cutter Cantor sets, in 'Fractal Geometry and Analysis', J. Bélair and S. Dubuc (eds.), Kluwer, (1991), 1-44, CMP 1140 719, Zbl 741.58011.

[B2] , Hausdorff dimension and box dimension for self-similar sets, Proc. Topology and Measure, V (1988), 17-26, Ernst-Moritz-Arndt Universität, MR 91a:58139, Zbl 743.54020.

[BU] T. Bedford and M. Urbański, The box and Hausdorff dimension of self-affine sets, Ergod. Th. and Dynam. Sys., 10 (1990), 627-644, MR 92b:58125, Zbl 725.28006.

[Bo1] R. Bowen, Equilibrium states and the ergodic theory of Anosov diffeomorphisms, Springer Lecture Notes in Mathematics, 470, Springer, 1975, MR 56 \#1364, Zbl 308.28010.

[Bo2] , Hausdorff dimension of quasi-circles, Publ. Math. I.H.E.S., 50 (1979), 11-25, Zbl 439.30032

[F1] K. Falconer, The Hausdorff dimension of self-affine fractals, Math. Proc. Camb. Phil. Soc., 103 (1988), 339-350, MR 89h:28010, Zbl 642.28005.

[F2] Fractal Geometry: Mathematical Foundations and Applications, John Wiley and Sons, 1990.

[K] R. Kaufman, On Hausdorff dimension of projections, Mathematika, 15 (1968), 153-155, MR 40 \#2030, Zbl 165.37404.

[KSS] M. Keane, M. Smorodinsky and B. Solomyak, On the morphology of $\gamma$ expansions with deleted digits, Trans. Amer. Math. Soc., 347(3) (1995), 955-966, MR 95h:11079, Zbl 834.11033.

[LL] A. Lopes and S. Lopes, On the dimension of the predictive process of a memoryless channel, Stochastic Anal. Appl., 14(4) (1996), 411-420, MR 97k:60180, Zbl 874.60065.

[Ly] R. Lyons, Singularity of some random continued fractions, J. Theor. Prob., 13(2) (2000), 535-545, CMP 1778585.

[M] P. Mattila, Geometry of sets and measures in Euclidean spaces, C.U.P., Cambridge, 1995, MR 96h:28006, Zbl 819.28004 
[MU1] R.D. Mauldin and M. Urbański, Dimensions and measures in infinite iterated function systems, Proc. London Math. Soc., 73(3) (1996), 105-154, MR 97c:28020, Zbl 852.28005.

[MU2] _ Dimensions and measures for a curvilinear Sierpinski gasket or Apollonian packings, Adv. Math., 136 (1998), 26-38, MR 99h:28015.

[MU3] - Parabolic iterated function systems, Ergod. Th. Dynam. Sys., 20(5) (2000), 1423-1447, CMP 1786722.

[PS] Y. Peres and W. Schlag, Smoothness of projections, Bernoulli convolutions, and the dimension of exceptions, Duke Math. J., 102(2) (2000), 193-251, MR 2001d:42013.

[PSo1] Y. Peres and B. Solomyak, Absolute continuity of Bernoulli convolutions, a simple proof, Math. Research Letters, 3(2) (1996), 231-239, MR 97f:28006, Zbl 867.28001.

[PSo2] - Self-similar measures and intersections of Cantor sets, Trans. Amer. Math. Soc., 350(10) (1998), 4065-4087, MR 98m:26009, Zbl 912.28005.

[PSo3] _ Problems on self-simiar and self-affine sets, an update, in 'Fractals and Stochastics II, Proceedings of the Greifswald conference' (Aug 1998), (2000), 95-106. MR 2001e:28014, Zbl 946.28003.

[Pi] S. Pincus, Singular stationary measures are not always fractal, J. Theor. Prob., 7(1) (1994), 199-208, MR 94j:60128, Zbl 791.28007.

[PoS] M. Pollicott and K. Simon, The Hausdorff dimension of $\lambda$-expansions with deleted digits, Trans. Amer. Math. Soc., 347 (1995), 967-983, MR 95h:11080, Zbl 831.28005.

[PrU] F. Przytycki and M. Urbański, On the Hausdorff dimension of some fractal sets, Studia Mathematica, 93 (1989), 155-186, MR 90f:28006, Zbl 691.58029.

[R] D. Ruelle, Repellers for real analytic maps, Ergod. Th. Dynam. Sys., 2 (1982), 99-107, MR 84f:58095, Zbl 506.58024.

[Sc] F. Schweiger, Ergodic Theory and Fibred Systems and Metric Number Theory, O.U.P. Oxford, 1995, MR 97h:11083, Zbl 819.11027.

[Si1] K. Simon, Hausdorff dimension for non-invertible maps, Ergod. Th. Dynam. Sys., 13 (1993), 199-212, MR 94c:58146, Zbl 781.58022.

[Si2] The Hausdorff dimension of the general Smale-Williams solenoid, Proc. Amer. Math. Soc., 125(4) (1997), 1221-1228, MR 97f:58086, Zbl 865.58033.

[SSo] K. Simon and B. Solomyak, Hausdorff dimension for horseshoes in $\mathbb{R}^{3}$, Ergod. Th. Dynam. Sys., 9(5) (1999), 1343-1363, CMP 1721625.

[SSU2] K. Simon, B. Solomyak and M. Urbański, Invarant measures for parabolic IFS with overlaps, Trans. Amer. Math. Soc., 353 (2001), 5145-5164.

[So1] B. Solomyak, On the random series $\sum \pm \lambda^{i}$ (an Erdös problem), Annals of Math., 142 (1995), 611-625, MR 97d:11125, Zbl 837.28007.

[So2] - Measure and dimension for some fractal families, Math. Proc. Camb. Phil. Soc., Math. Proc. Cambridge Phil. Soc., 124(3) (1998), 531-546, MR 99e:28016, Zbl 927.28006. 
[U] M. Urbański, Parabolic Cantor sets, Fundamenta Math., 151 (1996), 241-277, MR 98f:58076, Zbl 895.58036.

Received November 16, 1999. The authors were supported in part by the OTKA foundation grant F019099 and NSF grants DMS 9800786 and DMS 9801583.

Department of Stochastics

INSTITUTE OF MATHEMATICS

TeChnical University of Budapes

1521 Budapest P.O.Box 91

HUNGARY

E-mail address: simonk@math.bme.hu

Box 354350, Department of Mathematics

UNIVERSITY OF WASHINGTON

Seattle, WA 98195-4350

E-mail address: solomyak@math.washington.edu

Department of Mathematics

University OF NoRTh TEXAS

DENTON, TX 76203-1430

E-mail address: urbanski@unt.edu 